CAMP Working Paper Series

No $1 / 2016$

\title{
Approximating time varying structural models with time invariant structures
}

Fabio Canova, Filippo Ferroni and Christian Matthes

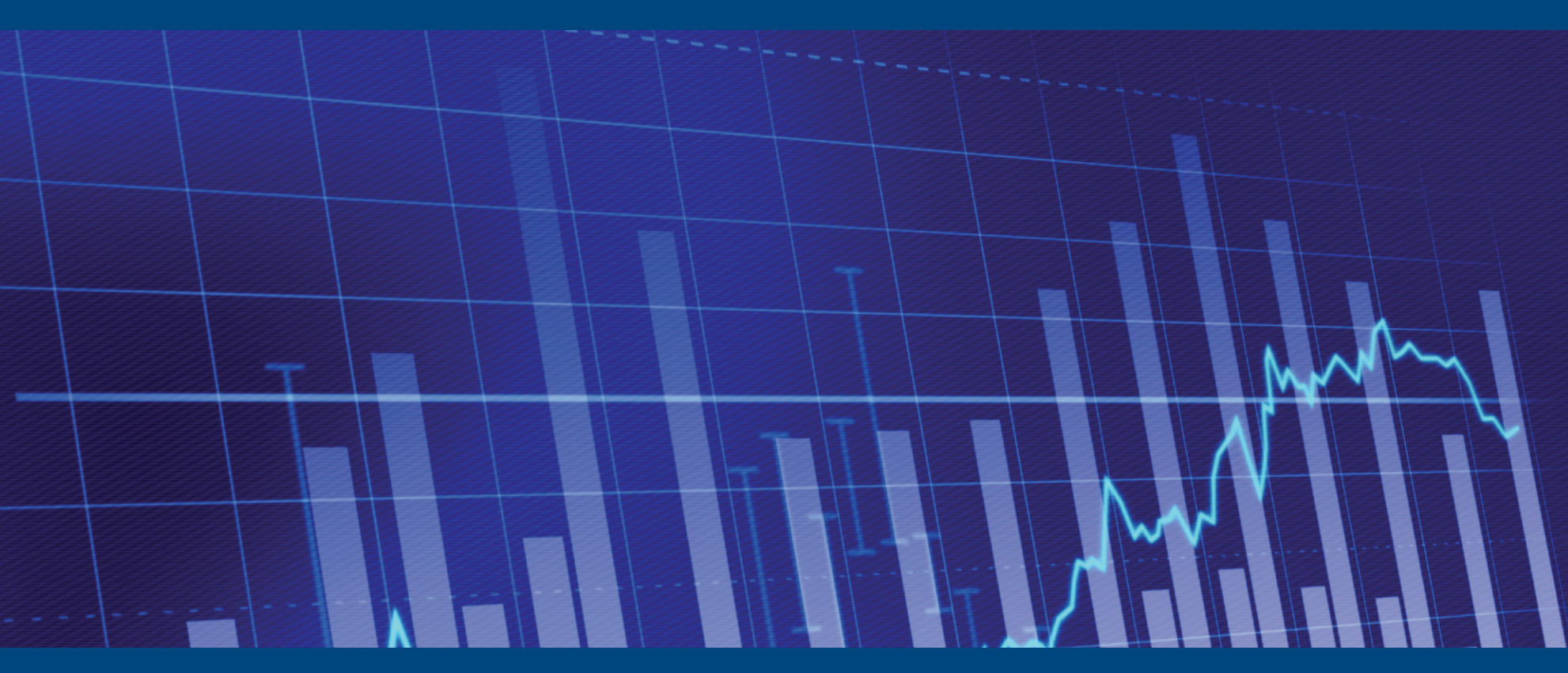

(C) Authors 2016

This paper can be downloaded without charge from the CAMP website http://www.bi.no/camp 


\title{
Approximating time varying structural models with time invariant structures
}

\author{
Fabio Canova, BI Norwegian Business School, CAMP, and CEPR \\ Filippo Ferroni Banque de France and University of Surrey \\ Christian Matthes Federal Reserve Bank of Richmond *
}

January 18, 2016

\begin{abstract}
The paper studies how parameter variation affects the decision rules of a DSGE model and structural inference. We provide diagnostics to detect parameter variations and to ascertain whether they are exogenous or endogenous. Identification and inferential distortions when a constant parameter model is incorrectly assumed are examined. Likelihood and VAR-based estimates of the structural dynamics when parameter variations are neglected are compared. Time variations in the financial frictions of Gertler and Karadi's (2010) model are studied.
\end{abstract}

Key words: Structural model, time varying coefficients, endogenous variations, misspecification.

JEL Classification: C10, E27, E32.

${ }^{*}$ We thank Michele Lenza, Marco del Negro, Tao Zha, Ferre de Graeve, James Hamilton, Frank Schorfheide, and the participants to seminars at Goethe University, University of Milan, Bank of England, Carlos III Madrid, Humboldt University Berlin, Federal Reserve Board, and the conferences ESSIM 2015; Identification in Macroeconomics, National Bank of Poland; Econometric Methods for business cycle analysis, forecasting and policy simulations, Norges Bank; the NBER Summer Institute group on Dynamic equilibrium models for comments and suggestions. The views presented in this paper do not reflect those of the Banque de France, the Federal Reserve Bank of Richmond, or the Federal Reserve system. 


\section{Introduction}

In macroeconomics it is standard to study models that are structural in the sense of Hurwicz (1962); that is, models where the parameters characterizing the preference and the constraints of the agents and the technologies to produce goods and services are invariant to changes in the parameters describing government policies. Such a requirement is crucial to distinguish structural from reduced form models, and to conduct correctly designed policy counterfactuals in dynamic stochastic general equilibrium (DSGE) models.

Recently, Dueker et al. (2007), Fernandez Villaverde and Rubio Ramirez (2007), Canova (2009), Rios Rull and Santaeularia Llopis (2010), Liu et al. (2011), Galvao, et al. (2014), Vavra (2014), Seoane (2014), and Meier and Sprengler (2015) have shown that DSGE parameters are not time invariant and that variations display small but persistent patterns. Parameter variations can not be taken as direct evidence that DSGE models are not structural. For example, Cogley and Yagihashi (2010), and Chang et al. (2013) showed that parameter variations may result from the misspecification of a time invariant model, while Schmitt Grohe and Uribe (2003) indicated that parameter variations may be needed in certain small open economy models to ensure the existence of a stationary equilibrium.

The approach the DSGE literature has taken to model parameter variations follows the VAR literature (see Cogley and Sargent, 2005, and Primiceri, 2005): they are assumed to be exogenously drifting as independent random walks. Many economic questions, however, hint at the possibility that parameter variations may instead be endogenous. For example, is it reasonable to assume that a central bank reacts to inflation in the same way in an expansion or in a contraction? Davig and Leeper (2006) analyze state-dependent monetary policy rules and describe how this feature affects structural dynamics. Does the propagation of shocks depend on the state of private and government debt? Do fiscal multipliers depend on inequality, see e.g. Brinca et al. (2014)? Are households as risk averse or as impatient when they are wealthy as when they are poor? Questions of this type are potentially numerous. Clearly, policy analyses conducted assuming time invariant parameters or an inappropriate form of time variations may be misleading; comparisons of the welfare costs of business cycles biased; and growth prescriptions invalid.

This paper has three main goals. First, we want to characterize the decision rules of a DSGE when parameter variations are either exogenous or endogenous, and in the latter case, when agents internalize or not the effects that their decisions may have on parameter variations. Second, we wish to provide diagnostics to detect misspecifications due to neglected parameter variations. Third, we want to study the consequences in terms of identification, estimation, and inference of using time invariant models when the DGP features parameter variations and compare likelihood-based and SVAR-based estimates of the structural dynamics when parameter variations are neglected.

The existing literature is generally silent on these issues. Seoane (2014) uses 
parameter variations as a respecification tool. Kulish and Pagan (2014) characterize the decision rules of a DSGE model when predictable structural breaks occur. Magnusson and Mavroedis (2014) and Huang (2014) examine how variations in the certain parameters may affect the identification of other structural parameters and the asymptotic theory of maximum likelihood estimators. Fernandez Villaverde et al. (2013) investigate to what extent variations in shock volatility matter for real variables. Ireland (2007) assumes that trend inflation in a standard New Keynesian model is driven by structural shocks; Ascari and Sbordone (2014) highlight that it may be a function of policy decisions.

The next section characterizes the decision rules in a general setup where both exogenous and endogenous variations in the parameters regulating preferences, technologies, and constraints are possible. We consider both first order and higher order perturbed approximations. We present a simple $\mathrm{RBC}$ example to provide intuition for the results we obtain. We show that if parameter variations are exogenous, structural dynamics are the same as in a model with no parameter variations. Thus, if one correctly identifies structural disturbances, she would make no mistakes in characterizing structural impulse responses, even if she employes a constant coefficient model. Clearly, variance and historical decompositions exercises will be distorted, since some sources of disturbances will be omitted. If parameter variations are instead endogenous, structural dynamics may be different from those of a constant coefficient model. Differences exist because the income and substitution effects present in the constant coefficient model are altered. These conclusions do not necessarily hold when higher order approximations are used.

Section 3 provides diagnostics to detect misspecification induced by neglecting parameter variations and to distinguish exogenous vs. endogenous parameter variations. In the context of a Monte Carlo exercise, we show that they are able to detect the true DGP with high probability

In section 4 we are interested in measuring the identification repercussions that neglected time variations may have for time invariant parameters. Since the likelihood is constructed using forecast errors, which are generally misspecified when parameter variations are neglected, one expects the likelihood shape to be both flattened and distorted. In the context of the RBC example, we show that indeed both pathologies occur; we also show that weakly identified (time invariant) parameters do not become better identified when time variations in other parameters exist.

Section 5 considers structural estimation of a time invariant model when the data is generated by models with time varying parameters. We expect distortions because the dynamics assumed by the constant coefficient model are generally incorrect and because shock misaggregation is present. Indeed, important biases in parameter estimates are present, occur primarily in parameters controlling income and substitution effects, and do not die away as sample size increase. Estimated impulse responses differ from the true ones both in quantitative and qualitative sense.

Section 6 studies whether a less structural time invariant SVARs model can capture the dynamics induced by structural shocks. We show that the performance 
is comparable if not superior to the one of structural models. The performance of SVARs worsens when shocks to the parameters account for a considerable portion of the variability of the endogenous variables but the deterioration is not as large as with likelihood -based approaches.

Section 7 estimates the parameters of Gertler and Karadi's (2010) model of unconventional monetary policy, applies the diagnostics to detect parameter variations, and estimates versions of the model where the bank's moral hazard parameter is allowed to vary over time. We find that a fixed coefficient model is misspecified, that making parameter variations endogenous function of net worth is preferable, and that the dynamic effects of capital quality shocks on the spread and on bank net worth can be more persistent than previously thought. Section 8 concludes.

\section{The setup}

The optimality conditions of a DSGE model can be represented as:

$$
E_{t}\left[f\left(X_{t+1}, X_{t}, X_{t-1}, Z_{t+1}, Z_{t}, \Theta_{t+1}, \Theta_{t}\right)\right]=0
$$

where $X_{t}$ is an $n_{x} \times 1$ vector of endogenous variables, $Z_{t}$ is an $n_{z} \times 1$ vector of strictly exogenous variables, $\Theta_{t}=\left[\Theta_{1 t}, \Theta_{2 t}\right]$, vector of possibly time varying structural parameters, where $\Theta_{2 t}$ is a $n_{\theta_{1}} n_{x_{1}} \times 1$ vector, $n_{x} \geq n_{x_{1}}$, appearing in the case agents internalize the effects that their decisions have on the parameters and $\Theta_{1 t}$ is an $n_{\theta_{1}} \times 1$ vector, while $f$ is a continuous function, assumed to be differentiable up to order $\mathrm{q}$, mapping onto a $\mathbb{R}^{n_{x}}$ space. Since the distinction between variables and parameters is blurred when we allow for parameter variations, we use the convention that parameters are those variables that typically assumed to be constant by economists.

The law of motion of the exogenous variables is:

$$
Z_{t+1}=\Psi\left(Z_{t}, \sigma \Sigma_{\epsilon} \epsilon_{t+1}^{z}\right)
$$

where $\Psi$ is a continuous function, assumed to be differentiable up to order q, mapping onto a $\mathbb{R}^{n_{z}}$ space; $\epsilon_{t+1}^{z}$ is a $n_{e} \times 1$ vector of i.i.d. structural disturbances with mean zero and identity covariance matrix, $n_{z} \geq n_{e} ; \sigma \geq 0$ is an auxiliary scalar; $\Sigma_{\epsilon}$ is a known $n_{e} \times n_{e}$ matrix. The law of motion of the structural parameters is:

$$
\Theta_{t+1}=\Phi\left(\Theta, X_{t}, U_{t+1}\right)
$$

where $\Phi$ is a continuous function, assumed to be differentiable up to order q, mapping onto the $\mathbb{R}^{n_{\theta}}$ space; $U_{t}$ is a $n_{u} \times 1$ vector of exogenous disturbances, $n_{\theta}=n_{\theta_{1}}\left(1+n_{x_{1}}\right) \geq$ $n_{u} ; \Theta$ is a vector of constants. The law of motion of $U_{t+1}$ is:

$$
U_{t+1}=\Omega\left(U_{t}, \sigma \Sigma_{u} \epsilon_{t+1}^{u}\right)
$$

where $\Omega$ is continuous and differentiable up to order q, mapping onto the $\mathbb{R}^{n_{u}}$ space; $\epsilon_{t}^{u}$ is a $n_{u} \times 1$ vector of i.i.d. disturbances, with mean zero and identity covariance matrix, uncorrelated with the $\epsilon_{t+1}^{z}$, and $\Sigma_{u}$ is a known $n_{u} \times n_{u}$ matrix. 
The decision rule is assumed to be of the form:

$$
X_{t}=h\left(X_{t-1}, W_{t}, \sigma \Sigma \epsilon_{t+1}, \Theta\right)
$$

where $h$ is a continuous function, assumed to be differentiable up to order $\mathrm{q}$, and mapping onto a $\mathbb{R}^{n_{x}}$ space, $\epsilon_{t+1}=\left[\epsilon_{t+1}^{z \prime}, \epsilon_{t+1}^{u \prime}\right]^{\prime}, \Sigma=\operatorname{diag}\left[\Sigma_{z}, \Sigma_{u}\right], W_{t}=\left[Z_{t}^{\prime}, U_{t}^{\prime}\right]^{\prime}$.

Few features of the setup need some discussion. First, $\Theta_{t}$ will be serially correlated if $U_{t}$ or $X_{t}$ or both are serially correlated. Second, the vector of structural disturbances $\epsilon_{t+1}^{z}$ may be smaller than the vector of exogenous variables and the dimension of $\epsilon_{t+1}^{u}$ may be smaller than the dimension of the structural parameters. Thus, there may be common patterns of variations in $Z_{t+1}$ and $U_{t+1}$. Third, we allow for time variations in the parameters regulating preferences, technologies, and constraints, but we do not consider variations in the auxiliary parameters regulating the law of motion of $Z_{t}$ and $U_{t}$, as we are not interested in stochastic volatility, GARCH, or rare events phenomena (as in e.g. Andreasan, 2012), nor in time variations driven by evolving persistence of the exogenous processes. Fourth, (5) makes no distinction between states and controls. Thus, it has the format of a final form (endogenous variables as a function of the exogenous variables and the parameters) rather than of a state space form (control variables as a function of the states and of the parameters).

\subsection{First order approximate decision rule}

We start by studying the implications of structural parameters variation for the optimal decision rule when a first order approximate solution is considered. Taking a linear expansion of (1) around the steady states leads to

$$
0=E_{t}\left[F x_{t+1}+G x_{t}+H x_{t-1}+L z_{t+1}+M z_{t}+N \theta_{t+1}+O \theta_{t}\right]
$$

where $F=\partial f / \partial X_{t+1}, G=\partial f / \partial X_{t}, H=\partial f / \partial X_{t-1}, L=\partial f / \partial Z_{t+1}, M=\partial f / \partial Z_{t}$, $N=\partial f / \partial \Theta_{t+1} O=\partial f / \partial \Theta_{t}$, all evaluated at the steady states values of $\left(X_{t}, Z_{t}, \Theta_{t}\right)$ and lower case letters indicate deviations from the steady states. Linear expanding (5) leads to:

$$
x_{t}=P x_{t-1}+Q z_{t}+R u_{t}
$$

where $P=\partial h / \partial X_{t-1}, Q=\partial h / \partial Z_{t}, R=\partial h / \partial U_{t}$, all evaluated at steady state values.

Proposition 2.1. The matrices $P, Q, R$ satisfy:

- $P$ solves $F P^{2}+\left(G+N \phi_{x}\right) P+\left(H+O \phi_{x}\right)=0$.

- Given $P, Q$ solves $V Q=-v e c\left(L \psi_{z}+M\right)$ and $V=\psi_{z}^{\prime} \otimes F+I_{n_{z}} \otimes\left(F P+G+N \phi_{x}\right)$.

- Given $P, R$ solves $W R=-\operatorname{vec}\left(N \phi_{u} \omega_{u}+O \phi_{u}\right)$ where $W=\omega_{u}^{\prime} \otimes F+I_{n_{\theta}} \otimes(F P+$ $\left.G+N \phi_{x}\right)$

where $\phi_{u}=\partial \Phi / \partial U_{t+1}, \phi_{x}=\partial \Phi / \partial X_{t}, \psi_{z}=\partial \Psi / \partial Z_{t}, \omega_{u}=\partial \Omega / \partial U_{t}$, vec denotes the columnwise vectorization, and where we assume that all the eigenvalues of $\psi_{z}$ and of $\omega_{u}$ are strictly less than one in absolute value. 
Proof. The proof is straightforward. Substituting (7) into (6), we obtain

$$
\begin{aligned}
0= & {\left[F P^{2}+\left(G+N \phi_{x}\right) P+\left(H+O \phi_{x}\right)\right] x_{t-1}+\left[\left(F P+G+N \phi_{x}\right) Q+F Q \psi_{z}+L \psi_{z}+M\right] z_{t} } \\
& +\left[\left(F P+G+N \phi_{x}\right) R+F R \omega_{u}+N \phi_{u} \omega_{u}+O \phi_{u}\right] u_{t}
\end{aligned}
$$

Since the solution must hold for every realization of $x_{t-1}, z_{t}, u_{t}$, we need to equate their coefficient to zero and the result obtains.

Corollary 2.2. If $\phi_{x}=0$, the dynamics in response to the structural shocks $z_{t}$ are identical to those obtained when parameters are time invariant. Variations in the $j$-th parameter have instantaneous impact on the endogenous variables $x_{t}$, if and only if the $j^{\text {th }}$ column of $N \phi_{u} \omega_{u}+O \phi_{u} \neq 0$.

Corollary 2.3. If $\phi_{u}=0$ and the matrices $N \phi_{x}$ and $O \phi_{x}$ are zero, parameter variations have no effects on the endogenous variables $x_{t}$.

Proposition 2.1 indicates that the first order approximate decision rule will, as in a constant coefficient setup, be a $\operatorname{VARMA}(1,1)$ but with an additional set of disturbances. Corollaries 2.2 and 2.3 give conditions under which parameter variations alter the dynamics induced by structural disturbances. If parameter variations are purely exogenous, $\phi_{x}=0$, the $\mathrm{P}$ and $\mathrm{Q}$ matrices are identical to those of a constant coefficient model. Thus, parameter variation adds variability to the endogenous variables without altering the dynamics produced by structural disturbances. In other words, suppose an economy is perturbed by technology shocks. Then, the dynamics induced by these shocks do not depend on whether the discount factor is constant or time varying, provided technological innovations are exogenous and unrelated to the innovations in the discount factor.

This result implies that if one is able to identify the structural disturbances $\epsilon_{t}^{z}$ from a time invariant version of the model, she would make no mistakes in characterizing structural dynamics. Clearly, variance or historical decomposition exercises will be distorted, since certain sources of variations (the $\epsilon_{t}^{u}$ disturbances) are omitted. One interesting question is whether standard procedures allow a researcher employing a time invariant model to recover $\epsilon_{t}^{z}$ from the data when the DGP features time varying structural parameters. If not, one would like to know which structural disturbance absorbs the missing shocks. Sections 5 and 6 study these issues in a practical example.

On the other hand, if parameter variations are purely endogenous, $\phi_{u}=0$, the dynamics in response to structural shocks may be altered. To know if distortions are present; one needs to check whether the columns of the matrices $N \phi_{x}$ and $O \phi_{x}$ are equal to zero. If they are not, a researcher employing a time invariant model is likely to incorrectly characterize both the structural dynamics and the relative importance of different sources of disturbances for the variability of the endogenous variables.

The equilibrium dynamics, as encoded in the $\mathrm{P}$ matrix, can thus help us to distinguish between models with endogenous time variation featuring different laws of motion for the parameters (i.e. the $\phi_{x}$ matrix). Distinguishing between models with 
exogenous time variation that differ in how the parameters respond to exogenous disturbances (i.e. the $\phi_{u}$ matrix) is possible if the cross equation restrictions present in $\mathrm{R}$ are different across models.

\subsection{Higher order approximate decision rule}

Are the conclusions maintained when higher order approximations are considered? In the second order approximation, the first order terms are the same as in the linear approximation. To examine whether quadratic terms will be affected by the presence of time variations insert (5) in the optimality conditions so that (1) is

$$
0=E_{t}\left[F\left(X_{t}, W_{t}, \sigma \Sigma \epsilon_{t+1}, \Theta\right)\right]
$$

The second order approximation of $(8)$ is

$$
\begin{aligned}
E_{t}\left[\left(F_{x} x_{t-1}+F_{w} w_{t}+F_{\sigma} \sigma\right)+0.5\left(F_{x x}\left(x_{t-1} \otimes x_{t-1}\right)+F_{w w}\left(w_{t} \otimes w_{t}\right)+F_{\sigma \sigma} \sigma^{2}\right)\right. & + \\
\left.F_{x w}\left(x_{t-1} \otimes w_{t}\right)+F_{x \sigma} x_{t-1} \sigma+F_{w \sigma} w_{t} \sigma\right] & =(9)
\end{aligned}
$$

Note that $F_{\sigma} \sigma, F_{x \sigma} x_{t-1} \sigma, F_{w \sigma} w_{t} \sigma$ are all zero, see Schmitt Grohe and Uribe (2004). The second order expansion of (5) is

$$
\begin{aligned}
x_{t} & =h_{x} x_{t-1}+h_{w} w_{t}+0.5\left(h_{x x}\left(x_{t-1} \otimes x_{t-1}\right)+h_{w w}\left(w_{t} \otimes w_{t}\right)+h_{\sigma \sigma} \sigma^{2}\right) \\
& +h_{x w}\left(x_{t-1} \otimes w_{t}\right)+h_{x \sigma} x_{t-1} \sigma+h_{w \sigma} w_{t} \sigma
\end{aligned}
$$

It is hard to make general statements about the properties of second order solutions

of models with time varying coefficients. As long as $F_{x x}, F_{w w}, F_{w x}$ are not affected by parameter variations, as is the case when variations are exogenous, second order approximations in time varying coefficient and in fixed coefficient models will be the same. However, when these expressions are affected, the approximations will be different. As an example of this latter case, consider the model

$$
\begin{aligned}
E_{t} y_{t+1} & =\theta_{t} x_{t}^{0.95} \\
x_{t} & =0.8 * x_{t-1}+0.2 * \bar{x}+u_{t} \\
\theta_{t} & =\left(2-0.5 *\left(\operatorname { e x p } \left(-\psi_{1}\left(x_{t-1}-\bar{x}\right)+\exp \left(\psi_{2}\left(x_{t-1}-\bar{x}\right)\right)+v_{t}\right.\right.\right.
\end{aligned}
$$

where both $v_{t}$ and $u_{t}$ are i.i.d. and $\bar{x} \equiv E x_{t}=1$. It is easy to verify that when $\psi_{1}=\psi_{2}$, the first order solution (including only the terms concerning structural dynamics) is

$$
y_{t}=0.76 x_{t-1}+0.95 u_{t}
$$

and it is the same as in the constant coefficient model $\left(\psi_{1}=\psi_{2}=0, v_{t}=0, \forall t\right)$, since $N \phi_{x}$ and $O \phi_{x}$ are both zero. However, the second order solution (including only terms concerning structural dynamics) is

$$
y_{t}=0.76 x_{t-1}+0.95 u_{t}-0.01565 x_{t-1}^{2}-0.2375 u_{t}^{2}+0.038 x_{t-1} u_{t}
$$


while the second order solution of the constant coefficient model is

$$
y_{t}=0.76 x_{t-1}+0.95 u_{t}-0.01520 x_{t-1}^{2}-0.2375 u_{t}^{2}-0.038 x_{t-1} u_{t}
$$

The $h_{x x}$ matrix differs in the two cases because, in general, $v_{t}$ may affect $y_{t+1}$ and thus alter higher order derivatives.

For higher order approximate solutions, the dynamics induced by structural shocks in constant coefficient and time varying coefficient models will generally differ, even with exogenous time variations. For example, in a third order approximation, the optimality conditions will feature terms in $F_{x \sigma \sigma}$ and $F_{w \sigma \sigma}$, which require a correction of the linear terms to account for uncertainty. Since in the constant coefficient model some shocks are omitted, one should expect the correction terms to differ in constant and time varying coefficient models.

\subsection{Discussion}

The results we derived require parameter variations to be continuous and smooth. This is in line with the evidence produced by Stock and Watson (1996) and with the standard practice employed in time varying coefficient VAR. Our framework is flexible and can accommodate once-and-for-all breaks (at a known date), as long as the transition between states is smooth. For example, a smooth threshold exogenously switching specification can be approximated with $\theta_{t+1}=(1-\rho) \theta+\rho \theta_{t}+a \exp (t-$ $\left.T_{0}\right) /\left(b+\exp \left(t-T_{0}\right)\right), t=1, \ldots, T_{0}-1, T_{0}, T_{0}+1, \ldots T$, where a and $\mathrm{b}$ are vectors, while $\theta_{t+1}=(1-\rho) \theta+\rho \theta_{t}+a \exp \left(-\left(X_{t}-X\right)\right) /\left(b+\exp \left(-\left(X_{t}-X\right)\right)\right.$, where $\mathrm{X}$ is the steady state value of $X_{t}$, can approximate smooth threshold endogenously switching specifications. What the framework does not allow for are Markov switching variations, occurring at unknown dates, as in Liu, et al. (2011), or abrupt changes, as in Davig and Leeper (2006), since the smoothness conditions on the $f$ function may be violated. Note, however, that our model becomes a close approximation to a Markov switching setup when the number of states is large.

It is important to emphasize that the (linear) solution we derive is a standard VAR with fixed coefficients and additional shocks. Thus, DSGE models with time varying coefficients do not generate new issues for aggregation or non-fundamentalness relative to a fixed coefficient DSGE model. More importantly, it is incorrect to consider time varying coefficient VAR as the reduced form counterpart of continuously varying coefficient DSGE models. One can show that there exists a state space representation of the solution where the (exogenously) time varying coefficients play the role of additional states of the model. What Proposition 2.1 shows is that the state space representation can be solved out to produce a standard VAR representation for the endogenous variables. Moreover, the proposition indicates that the matrices $\mathrm{P}$ and $\mathrm{Q}$ will be time varying only if $\phi_{x}$ is itself time varying. Thus, to match the time varying coefficient VAR evidence, it is necessary to consider variations in DSGE auxiliary parameters rather than variations in DSGE structural parameters.

Kulish and Pagan (2014) have developed solution and estimation procedures for models with abrupt breaks and learning between the states. Their solution for 
the pre-break and post-break period is a constant coefficient VAR, while for the learning period is a time varying coefficient VAR. Thus, a few words distinguishing the two approaches are needed. First, they are interested in characterizing the solution during the learning period, when the structure is unchanged, while we are interested in the decision rule when parameters are continuously varying. Second, their modelling of time variations is abrupt and the solution is designed to deal with that situation. Third, in our setup expectations are varying with the structure; in Kulish and Pagan they vary only in anticipation of a (foreseeable) break.

An alternative way of modelling time variations in (3) would be to make parameters functions of the exogenous rather than the endogenous variables, $\Theta_{t+1}=\Phi\left(\Theta, Z_{t}, U_{t+1}\right)$ as, for example, in Ireland (2007). While the equations the coefficients of the decision rule solve are different, the conclusions we have derived are unchanged by this modification. For, example, in the first order approximation, $\mathrm{P}$ now solves $F P^{2}+G P+H=0$; given $\mathrm{P}$, Q solves $V Q=-v e c\left(L \psi_{z}+M+N \phi_{z} \psi_{z}+O \phi_{z}\right)$ and $V=I_{n_{z}} \otimes\left(F P+G+F \phi_{z}\right)$; and given $\mathrm{P}, \mathrm{R}$ solves $W R=-v e c\left(N \phi_{u} \omega_{u}+O \phi_{u}\right)$, where $W=I_{n_{z}} \otimes\left(F P+G+F \omega_{u}\right)$.

While there are obvious economic differences between exogenous vs. endogenous coefficient variations, an alternative (statistical) way to think about the two specifications is that in the former each parameter evolves independently and covariations, if they exist, can be modelled by selecting the matrix $\Sigma_{u}$ to be of reduced rank. With endogenous variations, instead a set of observable factors (the $X$ 's) drives common parameter variations. Thus, $\Sigma_{u}$ is diagonal and full rank, unless some parameter variations are purely endogenous.

As (7) makes clear, it is hard to distinguish models with time varying coefficients from time invariant models with an additional set of shocks. In fact, models with $n_{1}$ structural shocks and $n_{2}$ time varying parameters, models with $n=n_{1}+n_{2}$ structural shocks and models with $n_{1}$ structural shocks and $n_{2}$ measurement errors are observationally equivalent:

$$
\begin{aligned}
x_{t} & =P x_{t-1}+Q z_{t}+R u_{t} \\
& =P x_{t-1}+Q^{*} z_{t}^{*} \\
& =P x_{t-1}+Q z_{t}+v_{t}
\end{aligned}
$$

where $Q^{*}=[Q, R] ; z_{t}^{*}=\left[z_{t}^{\prime}, u_{t}^{\prime}\right]^{\prime}, v_{t}=R u_{t}$. Thus, when designing time variation diagnostics, one must rule out a-priori all these potentially observational equivalent structures. In applications, procedures like the one described in section 3 or the one of Seoane (2014) can be used to select the interpretation of the additional shocks.

Finally, it is useful to compare the (linear) solution we derive with the solution obtained when coefficients are constant but the volatility of the shocks is stochastic. Neglecting second order terms, the solution in this latter case is $x_{t}=P x_{t-1}+Q z_{t}+A \sigma_{t}^{2}$. Thus, in empirical applications, it is crucial to allow for stochastic volatility to avoid to misrepresent volatility changes for parameter variations - a point made earlier by Sims (2001). 


\subsection{An example}

To convey some intuition into the mechanics of corollaries 2.2-2.3, we use a simple, closed economy, RBC model. The representative agent maximizes

$$
\max E_{0} \sum_{t=1}^{\infty} \beta_{t}\left(\frac{C_{t}^{1-\eta}}{1-\eta}-A \frac{N_{t}^{1+\gamma}}{1+\gamma}\right)
$$

subject to the sequence of constraints

$$
\begin{aligned}
Y_{t}\left(1-g_{t}\right) & =C_{t}+K_{t}-\left(1-\delta_{t}\right) K_{t-1} \\
Y_{t} & =\zeta_{t} K_{t-1}^{\alpha} N_{t}^{1-\alpha}
\end{aligned}
$$

where $Y_{t}$ is output, $C_{t}$ consumption, $K_{t}$ the stock of capital, $N_{t}$ is hours worked, and $g_{t}=\frac{G_{t}}{Y_{t}}$ is the share of government expenditure in output. The system is perturbed by two exogenous structural disturbances: one to the technology $Z_{t}$, and one to the government spending share, $g_{t}$, both assumed to follow time invariant $\mathrm{AR}(1)$ processes

$$
\begin{aligned}
& \ln \zeta_{t}=\left(1-\rho_{\zeta}\right) \ln \zeta+\rho_{\zeta} \ln \zeta_{t-1}+e_{t}^{\zeta} \\
& \ln g_{t}=\left(1-\rho_{g}\right) \ln g+\rho_{g} \ln g_{t-1}+e_{t}^{g}
\end{aligned}
$$

where variables without time subscript denote steady state quantities. There are 12 parameters in the model: 6 structural ones ( $\alpha$ is the capital share, $\eta$ the risk aversion coefficient, $\gamma$ the inverse of the Frisch elasticity of labor supply, A the constant in front of labor in utility, $\beta_{t}$ the time discount factor, and $\delta_{t}$ the depreciation rate), and 6 auxiliary ones (the steady state values of the government expenditure share and of TFP, $(\zeta, g)$, their autoregressive parameters, $\left(\rho_{\zeta}, \rho_{g}\right)$, and their standard deviations $\left.\left(\sigma_{\zeta}, \sigma_{g}\right)\right)$. We assume that all parameters but $\beta_{t}$ and $\delta_{t}$ are time invariant. Dueker et al. (2007), Liu et al (2011), Gourio (2012), Karabarbounis and Neiman (2014), and Meier and Sprenger (2015) provide evidence that these parameters are indeed evolving over time. The first order approximation to the law of motion of $\left(\beta_{t}, \delta_{t}\right)$ is described below.

The optimality conditions of the problem are:

$$
\begin{aligned}
A C_{t}^{\eta} N_{t}^{\gamma} & =(1-\alpha)\left(1-g_{t}\right) Y_{t} / N_{t} \\
\beta_{t} C_{t}^{-\eta} & =E_{t}\left(\beta_{t+1} C_{t+1}^{-\eta}\left(\frac{\alpha\left(1-g_{t+1}\right) Y_{t+1}}{K_{t+1}}+1-\delta_{t+1}\right)\right. \\
& \left.+E_{t}\left(\frac{\partial \beta_{t+1}}{\partial K_{t}} u\left(C_{t+1}, N_{t+1}\right)-\frac{\partial \delta_{t+1}}{\partial K_{t}} K_{t}\right)\right) \\
\left(1-g_{t}\right) Y_{t} & =C_{t}+K_{t}-\left(1-\delta_{t}\right) K_{t-1} \\
Y_{t} & =\zeta_{t} K_{t-1}^{\alpha} N_{t}^{1-\alpha}
\end{aligned}
$$

Time variations in $\beta_{t}$ and $\delta_{t}$ affect optimal choices in two ways. There is a direct effect in the Euler equation and in the resource constraint when $\beta_{t}$ and $\delta_{t}$ are time 
varying; and if agents take into account that their decisions may affect parameter variations, there will be a second (endogenous) effect due variations in the derivatives of $\beta_{t+1}$ and $\delta_{t+1}$ with respect to the endogenous states - see equation (23).

Note that varying parameters can not be considered wedges in the sense of Chari et al. (2007), because there are cross-equation restrictions that need to be satisfied. Furthermore, while the rank of the covariance matrix of the wedges is full, this is not necessarily the case in our setup.

We specialize this setup to consider various possibilities.

\subsubsection{Model A: Constant coefficients.}

As a benchmark, we let $\beta_{t}=\beta^{t}$ and $\delta_{t}=\delta$. The optimality conditions are

$$
E_{t}\left(\begin{array}{c}
E_{t}\left[f\left(X_{t+1}, X_{t}, X_{t-1}, Z_{t+1}, Z_{t}, \Theta\right)\right]= \\
A C_{t}^{\eta} N_{t}^{\gamma+1}-(1-\alpha)\left(1-g_{t}\right) Y_{t} \\
C_{t}^{-\eta}-E_{t} \beta C_{t+1}^{-\eta}\left(\alpha\left(1-g_{t+1}\right) Y_{t+1} / K_{t}+1-\delta\right) \\
\left(1-g_{t}\right) Y_{t}-C_{t}+K_{t}-(1-\delta) K_{t-1} \\
Y_{t}-\zeta_{t} K_{t-1}^{\alpha} N_{t}^{1-\alpha}
\end{array}\right)=0
$$

$X_{t}=\left(K_{t}, Y_{t}, C_{t}, N_{t}\right)^{\prime}, Z_{t}=\left(\zeta_{t}, g_{t}\right)^{\prime}$. In the steady state, we have:

$$
\frac{K}{Y}=\frac{\alpha(1-g)}{\delta-1+1 / \beta} ; \frac{C}{Y}=1-\delta \frac{K}{Y}-\frac{g}{Y} ; \frac{N}{Y}=\zeta^{\frac{1}{1-\alpha}}\left(\frac{K}{Y}\right)^{\frac{\alpha}{\alpha-1}} ; Y=\left[\frac{A}{(1-\alpha)(1-g)}\left(\frac{C}{Y}\right)^{\eta}\left(\frac{N}{Y}\right)^{1+\gamma}\right]^{-\frac{1}{\eta+\gamma}}
$$

\subsubsection{Model B: Exogenous parameter variations}

Set $d_{t}=\beta_{t+1} / \beta_{t}$. We let $\Theta_{t+1}-\Theta \equiv\left(d_{t+1}-\left(1-\rho_{\beta}\right) \beta, \delta_{t+1}-\left(1-\rho_{\delta}\right) \delta\right)^{\prime}=U_{t+1}$ and postulate

$$
\begin{aligned}
& u_{d, t+1}=\rho_{d} u_{d, t}+e_{d, t+1} \\
& u_{\delta, t+1}=\rho_{\delta} u_{\delta, t}+e_{\delta, t+1}
\end{aligned}
$$

Since $\Theta_{t+1}$ is exogenous, $\partial \beta_{t+1} / \partial K_{t}=\partial \delta_{t+1} / \partial K_{t}=0$ and the $f$ function becomes

$$
E_{t}\left(\begin{array}{c}
E_{t}\left[f\left(X_{t+1}, X_{t}, X_{t-1}, Z_{t+1}, Z_{t}, \Theta_{t+1}, \Theta_{t}\right)\right]= \\
A C_{t}^{\eta} N_{t}^{\gamma+1}-(1-\alpha)\left(1-g_{t}\right) Y_{t} \\
1-d_{t} C_{t+1}^{-\eta} / C_{t}^{-\eta}\left(\alpha\left(1-g_{t+1}\right) Y_{t+1} / K_{t}+1-\delta_{t+1}\right) \\
\left(1-g_{t}\right) Y_{t}-C_{t}-K_{t}+\left(1-\delta_{t}\right) K_{t-1} \\
Y_{t}-\zeta_{t} K_{t-1}^{\alpha} N_{t}^{1-\alpha}
\end{array}\right)=0
$$

where $X_{t}=\left(K_{t}, Y_{t}, C_{t}, N_{t}\right)^{\prime}, Z_{t}=\left(\zeta_{t}, g_{t}\right)^{\prime}$ and $\Theta_{t}=\Theta_{1 t}$.

With the selected parameterization the steady state values of $\left(\frac{K}{Y}, \frac{C}{Y}, \frac{N}{Y}, Y\right)$ coincide with those of the constant coefficient model. In addition, since $\phi_{x}=0$, variations 
in $\left(d_{t+1}, \delta_{t+1}\right)$ leave the decision rule matrices $\mathrm{P}$ and $\mathrm{Q}$ as in model $\mathrm{A}$. Thus, as far as structural dynamics are concerned, models $\mathrm{A}$ and $\mathrm{B}$ are observationally equivalent.

To examine whether variations in $\Theta_{t}$ have an instantaneous impact on $X_{t}$, we need to check the columns of $N \phi_{u} \omega_{u}+O \phi_{u}$.

$$
N \phi_{u} \rho_{u}+O \phi_{u}=\left(\begin{array}{cc}
0 & 0 \\
-1 / \beta & -\rho_{\delta} / \beta \\
0 & -K \\
0 & 0
\end{array}\right) \neq 0
$$

Note that if $d_{t}$ were a fast moving variable, the impact effect on $X_{t}$ would depend on the persistence of shocks to the growth rate of the discount factor. For example, if $\rho_{d}=0$, shocks to the growth rate of the time discount factor have no effects on $X_{t}$. Thus, if only the discount factor is time varying and variations in its growth rate are i.i.d., models A and B have identical decision rules.

\subsubsection{Model C: Endogenous parameter variations, no internalization}

Assume that the time variations in the growth rate of the discount factor and in the depreciation rate are driven by the aggregate capital stock. We specify

$$
\Theta_{t+1}=\left[\Theta_{u}-\left(\Theta_{u}-\Theta_{l}\right) e^{-\phi_{a}\left(K_{t}-K\right)}\right]+\left[\Theta_{u}-\left(\Theta_{u}-\Theta_{l}\right) e^{\phi_{b}\left(K_{t}-K\right)}\right]+U_{\theta, t+1}
$$

where $\phi_{a}, \phi_{b}, \Theta_{u}, \Theta_{l}$ are vectors of parameters and $\mathrm{U}_{\theta, t+1}$ is a zero mean, i.i.d. vector of shocks. This specification is flexible and depending on the choice of $\phi^{\prime} s$, we can accommodate linear or quadratic relationships, which are symmetric or asymmetric. To ensure that models $\mathrm{C}$ and $\mathrm{A}$ have the same steady states, we set $\Theta_{l}=(\beta / 2, \delta / 2)$.

We assume that agents treat the capital stock appearing in (32) as an aggregate variable. This assumption is similar to the 'small $\mathrm{k}$-big k' situation encountered in standard rational expectations models or to the distinction between internal and external habit formation. Thus, agents' first order conditions do not take into account the fact that their optimal capital choice changes $d_{t}$ and $\delta_{t}$ so $\partial \beta_{t+1} / \partial K_{t}=\partial \delta_{t+1} / \partial K_{t}=0$ and the equilibrium conditions are then as in (30). Since the $f$ function is the same as in model $\mathrm{B}$, the matrices $N$ and $O$ are unchanged.

To examine whether parameter variations affect the matrices regulating structural dynamics note that

$$
\begin{aligned}
& N \phi_{x}=\left(\begin{array}{cc}
0 & 0 \\
0 & 1 / \beta \\
0 & 0 \\
0 & 0
\end{array}\right)\left(\begin{array}{cccc}
\left(d_{u}-\beta / 2\right)\left(\phi_{11}-\phi_{21}\right) & 0 & 0 & 0 \\
\left(\delta_{u}-\delta / 2\right)\left(\phi_{12}-\phi_{22}\right) & 0 & 0 & 0
\end{array}\right)
\end{aligned}
$$

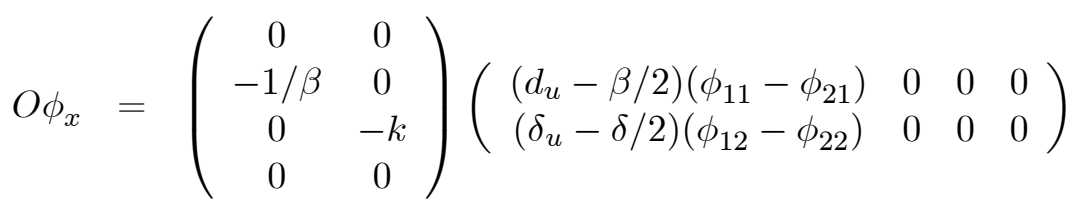


Endogenous variations in $d_{t}, \delta_{t}$ leave $\mathrm{P}$ and $\mathrm{Q}$ unaffected, unless $\phi_{1} \neq \phi_{2}$ and/or $\phi_{3} \neq$ $\phi_{4}$, i.e. unless there are asymmetries in the law of motion of $\left(d_{t}, \delta_{t}\right)$. To verify whether parameter variations impact on $\mathrm{X}_{t}$, check the columns of $N \phi_{u} \omega_{u}+O \phi_{u}$. We have:

$$
N \phi_{u} \omega_{u}+O \phi_{u}=\left(\begin{array}{cc}
0 & 0 \\
1 / \beta\left(d_{u}-\beta / 2\right)\left(-\phi_{1}+\phi_{2}\right) & 0 \\
0 & K\left(\delta_{u}-\delta / 2\right)\left(-\phi_{3}+\phi_{4}\right) \\
0 & 0
\end{array}\right) \neq 0
$$

if $\phi_{1} \neq \phi_{2}$, or $\phi_{3} \neq \phi_{4}$ and regardless of persistence of the shocks to the parameters.

\subsubsection{Model D: Endogenous parameter variations, internalization.}

We still assume that time variations in the discount factor and in the depreciation rate are driven by the aggregate capital stock and by an exogenous shock, as in equation (32). Contrary to case C, we assume that agents internalize the effects their capital decisions have on parameter variations. The relevant derivatives are

$$
\begin{aligned}
d_{t+1}^{\prime} & \equiv \partial d_{t+1} / \partial K_{t}=-\left(\beta_{u}-\beta / 2\right)\left[-\phi_{1} e^{-\phi_{1}\left(K_{t}-K\right)}+\phi_{2} e^{\phi_{2}\left(K_{t}-K\right)}\right] \\
\delta_{t+1}^{\prime} & \equiv \partial \delta_{t+1} / \partial K_{t}=-\left(\delta_{u}-\delta / 2\right)\left[-\phi_{3} e^{-\phi_{3}\left(K_{t}-K\right)}+\phi_{4} e^{\phi_{4}\left(K_{t}-K\right)}\right]
\end{aligned}
$$

In order for the steady states of model $\mathrm{D}$ to equal to those of model $\mathrm{A}$, we restrict $\phi_{1}=\phi_{2}=\phi_{1}, \phi_{3}=\phi_{4}=\phi_{3}$. The optimality conditions are:

$$
E_{t}\left(\begin{array}{c}
0=E_{t}\left[f\left(X_{t+1}, X_{t}, X_{t-1}, Z_{t+1}, Z_{t}, \Theta_{t+1}, \Theta_{t}\right)\right] \\
= \\
A C_{t}^{\eta} N_{t}^{\gamma+1}-(1-\alpha)\left(1-g_{t}\right) Y_{t} \\
u\left(d_{t+1}^{\prime}, N_{t+1}\right) / C_{t}^{-\eta}-d_{t} C_{t+1}^{-\eta} / C_{t}^{-\eta}\left(\alpha\left(1-g_{t+1}\right) Y_{t+1} / K_{t+1}+1-\delta_{t+1}+\delta_{t+1}^{\prime} K_{t}\right) \\
\left(1-g_{t}\right) Y_{t}-C_{t}-K_{t}+\left(1-\delta_{t}\right) K_{t-1} \\
Y_{t}-\zeta_{t} K_{t-1}^{\alpha} N_{t}^{1-\alpha}
\end{array}\right)
$$

where as before $X_{t}=\left(K_{t}, Y_{t}, C_{t}, N_{t}\right)^{\prime}, Z_{t}=\left(\zeta_{t}, g_{t}\right)^{\prime}$ but now $\Theta_{t}=\left(d_{t}, \delta_{t}, d_{t}^{\prime}, \delta_{t}^{\prime}\right)^{\prime}$ and

$$
\left(\begin{array}{c}
d_{t+1} \\
\delta_{t+1} \\
d_{t+1}^{\prime} \\
\delta_{t+1}^{\prime}
\end{array}\right)=\Phi\left(\Theta, K_{t}, U_{t+1}\right)=\left(\begin{array}{c}
2 d_{u}-\left(d_{u}-\beta / 2\right)\left[e^{-\phi_{1}\left(K_{t}-K\right)}+e^{\phi_{1}\left(K_{t}-K\right)}\right]+U_{\beta, t+1} \\
2 \delta_{u}-\left(\delta_{u}-\delta / 2\right)\left[e^{-\phi_{3}\left(K_{t}-K\right)}+e^{\phi_{3}\left(K_{t}-K\right)}\right]+U_{\delta, t+1} \\
-\left(d_{u}-\beta / 2\right) \phi\left[-e^{-\phi_{1}\left(K_{t}-K\right)}+e^{\phi_{1}\left(K_{t}-K\right)}\right] \\
-\left(\delta_{u}-\delta / 2\right) \phi\left[-e^{-\phi_{3}\left(K_{t}-K\right)}+e^{\phi_{3}\left(K_{t}-K\right)}\right]
\end{array}\right)
$$

The relevant matrices of derivatives evaluated at the steady states are $\omega_{u}=0_{2 \times 2}$,

$$
\begin{aligned}
& N=\frac{\partial f}{\partial \Theta_{t+1}}=\left(\begin{array}{cccc}
0 & 0 & 0 & 0 \\
0 & 1 / \beta & -u(C, N) / C^{-\eta} & -\beta K \\
0 & 0 & 0 & 0 \\
0 & 0 & 0 & 0
\end{array}\right), \quad O=\frac{\partial f}{\partial \Theta_{t}}=\left(\begin{array}{cccc}
0 & 0 & 0 & 0 \\
-1 / \beta & 0 & 0 & 0 \\
0 & -K & 0 & 0 \\
0 & 0 & 0 & 0
\end{array}\right) \\
& \phi_{x}=\left(\begin{array}{cccc}
0 & 0 & 0 & 0 \\
0 & 0 & 0 & 0 \\
-2\left(\beta_{u}-\beta / 2\right) \phi_{1}^{2} & 0 & 0 & 0 \\
-2\left(\delta_{u}-\delta / 2\right) \phi_{3}^{2} & 0 & 0 & 0
\end{array}\right), \quad \phi_{u}=\left(\begin{array}{cc}
0 & 0 \\
0 & 0 \\
-2\left(\beta_{u}-\beta / 2\right) \phi_{1}^{2} & 0 \\
0 & -2\left(\delta_{u}-\delta / 2\right) \phi_{3}^{2}
\end{array}\right)
\end{aligned}
$$


Clearly, $N \phi_{x} \neq 0$, and $N \phi_{u} \omega_{u}+O \phi_{u}=0$. Thus, a shock to the law of motion of the parameters alters the dynamics produced by structural shocks, even when the relationship between parameters and states is symmetric.

In sum, parameter variations matter for the structural dynamics either if the relationship between parameters and the states is asymmetric or if agents internalize the consequences their decisions have on parameter variations, or both.

\subsubsection{Impulse responses}

Why are structural dynamics in models $\mathrm{C}$ and $\mathrm{D}$ different from those in model A? To understand what drives economic differences, we compute impulse responses. For the parameters common to all models, we choose $\alpha=0.30, \beta=0.99, \delta=0.025$, $\gamma=2, \eta=2, A=4.50, \zeta=1 ; \rho_{\zeta}=0.90, \sigma_{\zeta}=0.00712, g=0.18, \rho_{g}=0.50$ and $\sigma_{g}=0.01$. For the other parameters, we choose:

- Model $B: \rho_{\beta}=0.985, \rho_{\delta}=0.95$ and $\sigma_{\beta}=0.002 \sigma_{\delta}=0.07$.

- Model $C: \phi_{1 \beta}=0.01, \phi_{2 \beta}=0.03, \phi_{1 \delta}=0.2, \phi_{2 \delta}=0.1, \sigma_{d}=\sigma_{\delta}=0.5, \beta_{u}=$ $0.999, \delta_{u}=0.025$.

- Model $D: \phi_{1 \beta}=0.0001, \phi_{2 \beta}=0.016, \phi_{1 \delta}=0.2, \phi_{2 \delta}=0.1, \sigma_{d}=0.0001 ; \sigma_{\delta}=0.1$, $\beta_{u}=0.999, \delta_{u}=0.025$.

Figure 1 reports the responses of hours, capital, consumption, and output to the two structural shocks in the four models. The first column has the responses to technology shocks; the second has the responses to government expenditure shocks ${ }^{1}$.

Note first, that the sign of the responses is unchanged by the presence of parameter variations. The responses of models $C$ and $\mathrm{D}$ differ from those of model A in the shape and the persistence of consumption and capital responses. Differences occur because income and substitution effects are different. For instance, in response to technology shocks, agents work and save less and consume more in models $C$ and D than in the constant coefficients model, while in response to government expenditure shocks, consumption falls more and capital falls less relative to the constant coefficients case. Thus, parameter variations play the same role as uncertainty variations and make agents desire to smooth less transitory structural shocks.

\footnotetext{
${ }^{1}$ Since the responses of hours and output to government expenditure shocks are different from what the conventional wisdom indicates, a few words of explanation are needed. In a standard RBC in response to government expenditure shocks, hours and output typically increase because of a wealth effect. However, here the shock affects the share of government expenditure in GDP. Thus, the positive wealth effect on labor supply is absent because government expenditure increase in exactly the same proportion as output, thus disincentivizing agents to try to increase private output.
} 

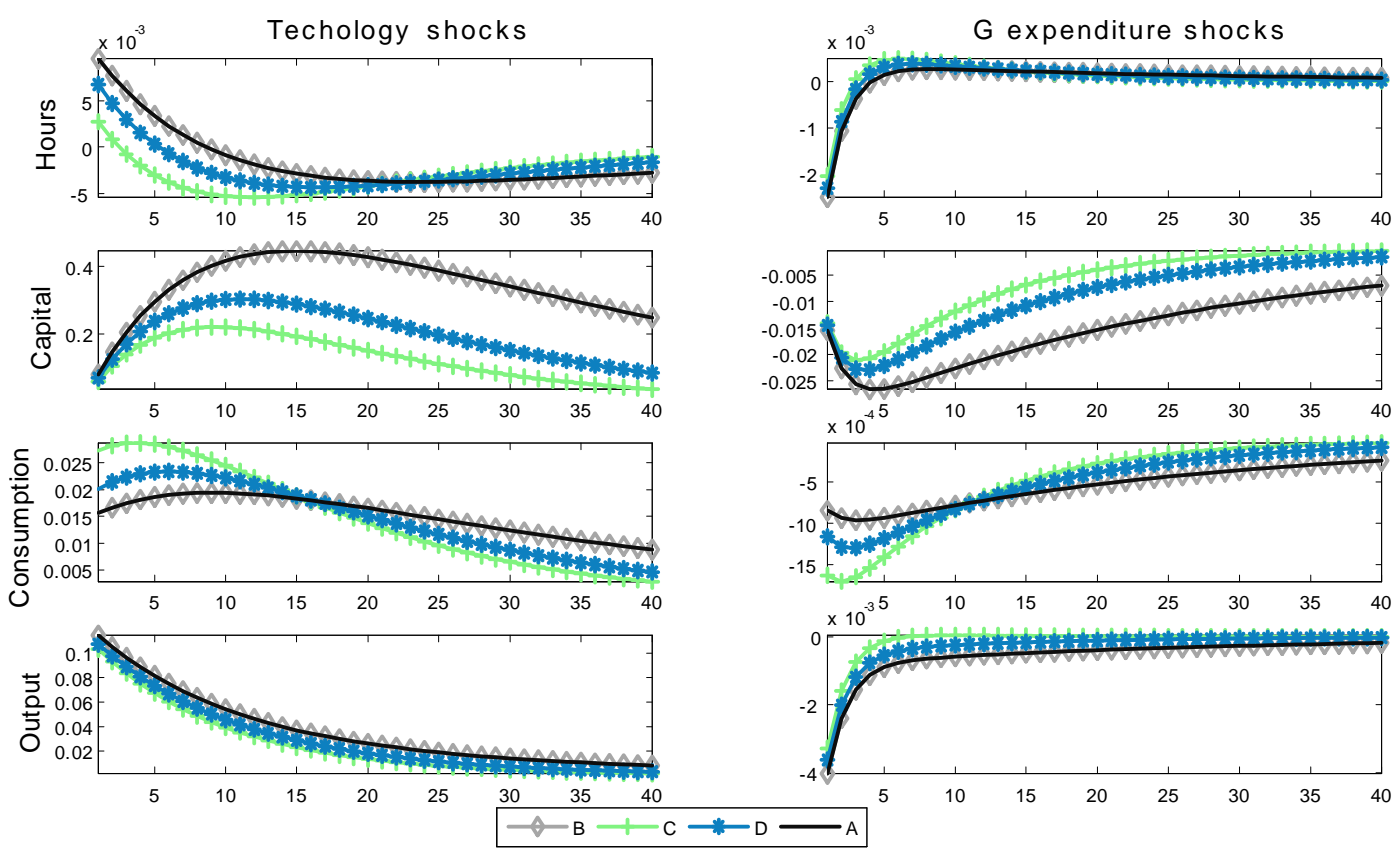

Figure 1: Impulse responses, first order approximation

\section{Characterizing time varying misspecification}

Because the decision rules of constant coefficient models are generally misspecified when the data generating process (DGP) features parameter variations, it is important to diagnose potential time varying problems. This section describes two diagnostics useful for the purpose: one based on "wedges" and one based on forecast errors.

Consider the optimality conditions of a constant coefficient model

$$
E_{t}\left[F\left(X_{t-1}, W_{t}, \sigma \Sigma \epsilon_{t+1}^{z}, \Theta\right)\right]=0
$$

obtained substituting for $X_{t}$ the decision rule:

$$
X_{t}=h\left(X_{t-1}, W_{t}, \sigma \Sigma \epsilon_{t+1}^{z}, \Theta\right)
$$

When $X_{t-1}$ has been generated by the constant coefficient model, $F$ is a martingale difference. When instead $X_{t-1}$ has been generated by a time varying coefficient model

$$
X_{t}^{*}=h^{*}\left(X_{t-1}^{*}, W_{t}, \sigma \Sigma \epsilon_{t+1}, \Theta\right)
$$

$\mathrm{E}\left[F\left(X_{t-1}^{*}, W_{t}, \sigma \Sigma \epsilon_{t+1}^{z}, \Theta\right)\right] \neq 0$, since $\sigma \Sigma \epsilon_{t+1}^{z} \neq \sigma \Sigma \epsilon_{t+1}$ and $\mathrm{h} \neq h^{*}$. Furthermore, $F\left(X_{t-1}^{*}, W_{t}, \sigma \Sigma \epsilon_{t+1}^{z}, \Theta\right)$ will be predictable using past values $X_{t-1}^{*}$. To see why, 
consider the first order approximate optimality conditions. In this system of equations, the wedge is

$$
\begin{array}{r}
\left(F\left(P^{*}-P\right)^{2}+G\left(P^{*}-P\right)\right) x_{t-1}^{*}+ \\
\left(F\left(Q^{*}-Q\right) \psi_{z}+G\left(Q^{*}-Q\right)+F\left(P^{*}-P\right)\left(G^{*}-G\right)\right) z_{t} \\
\left(F\left(P^{*}-P\right) R^{*}+G R^{*}+F R^{*} \omega_{u}\right) u_{t}
\end{array}
$$

When $P^{*}=P, Q^{*}=Q$, as in the exogenously varying model, the wedge reduces to

$$
\left(G R^{*}+F R^{*} \omega_{u}\right) u_{t}
$$

which differs from zero if $R^{*} \neq 0$ and will be predictable using $x_{t-j}^{*}, j \geq 1$, if $\omega_{u} \neq 0$. When, as in the endogenously varying model, $P^{*} \neq P, Q^{*} \neq Q$, the wedge will differ from zero, even when $R=0$, and will be predictable using past $x_{t-1}^{*}$, even when $\omega_{u}=0$.

Hence, to detect time varying misspecification, one can compute wedges and regress them on the lags of the observables. If they are significant, the martingale difference condition is violated, and there is evidence of time varying parameters. Note that the diagnostic uses the assumption that the model is correctly specified up to parameter variations. If the model is incorrect, lags of the observables may be significant, even without time varying coefficients. Inohue, Kuo, and Rossi (2015) apply this idea to detect generic model misspecification.

The logic of the forecast error diagnostic is similar. The linearized decision rule in a constant coefficients model is $x_{t}=P x_{t-1}+Q z_{t}$, while in a time varying coefficient model it is $x_{t}^{*}=P^{*} x_{t-1}^{*}+Q^{*} z_{t}+R^{*} u_{t}$. Let $v_{t}^{*}$ be the forecast error in predicting $x_{t}^{*}$ using the decision rules of the constant coefficient model and the data generated from the time varying coefficient model. The forecast error can be decomposed as

$$
v_{t}^{*}=x_{t}^{* j}-P x_{t-1}^{*}=Q^{*} z_{t}+R^{*} u_{t}+\left(P^{*}-P\right) x_{t-1}^{*}
$$

Thus, forecast errors are functions of the lags of the observables $x_{t-1}^{*}$ when $P^{*} \neq P$. However, even if $P^{*}=P$, forecasts error linearly depend on the lags of the observables if $u_{t}$ is serially correlated. Hence, an alternative way to check for parameter variations involves regressing the forecast errors $v_{t}^{*}$ on lagged values of the observables and checking the significance of the regression coefficients.

We apply the two diagnostics to 1,000 samples constructed using the RBC model previously considered. Table 1 reports the rejection rate of an F-statistic for the null hypothesis of no time variations at the 0.05 percent confidence level. The Euler wedge diagnostic has very good size properties (does not reject the hypothesis of no time variations) when the model has fixed coefficients; when it has fixed coefficients but it is locally misspecified - capacity utilization is neglected; and when the exogenous time variations are i.i.d.. It is somewhat conservative in detecting time variations when exogenous parameter variations are persistent and has excellent power properties when variations are endogenous. The forecast error diagnostic has good size properties when the DGP has no time variation and no misspecification is present but tends to overreject the null 


\begin{tabular}{|c|c|c|c|c|}
\hline DGP & \multicolumn{2}{|c|}{ Euler wedge } & \multicolumn{2}{c|}{ Forecast errors output } \\
\hline & \multicolumn{2}{|c|}{ F-test $c_{t-1}, r_{t-1}=0$} & \multicolumn{2}{c|}{ F-test $c_{t-1}, n_{t-1}, y_{t-1}=0$} \\
& $\mathrm{~T}=1000$ & $\mathrm{~T}=150$ & $\mathrm{~T}=1000$ & $\mathrm{~T}=150$ \\
\hline Fixed coefficients & 0.00 & 0.00 & 0.00 & 0.00 \\
Fixed coeff and capacity utilization & 0.001 & 0.003 & 1.00 & 0.98 \\
Exogenous TVC no serial correlation & 0.07 & 0.001 & 0.91 & 0.24 \\
Exogenous TVC & 0.53 & 0.40 & 1.00 & 0.90 \\
Endogenous TVC & 1.00 & 0.93 & 1.00 & 0.99 \\
\hline
\end{tabular}

Table 1: Percentage of rejections at the 0.05 confidence level of the null of no time variations in 1000 experiments. The dependent variable is either the Euler wedge or the forecast error in the output equation. The regressors are lagged consumption and interest rates for the Euler wedge; lagged output, consumption and hours for the forecast error.

if misspecification is present or exogenous time variations are i.i.d.. On the other hand, it has good power properties when time variations are present. Because of the differences they display, it seems wise to use both diagnostics in empirical applications.

\subsection{Exogenous vs. endogenous parameter variations}

If the diagnostics of the previous subsection indicate the presence of parameter variations, one may interested in knowing whether they are of exogenous or endogenous type. One way to distinguish the two options is to use the DGSE-VAR methodology of Del Negro and Schorfheide (2004). In a DSGE-VAR, one uses the DSGE model as a prior for the VAR of the observable data and employs the marginal likelihood to measure the value of the additional information the DSGE provides. If the additional observations come from the DGP, the quality of the estimates improves (standard errors are reduced), and the marginal likelihood increases. On the other hand, if the additional observations come from a DGP different from the one generating the data, biases may be introduced, noise added, and the precision of the estimates and the fit of the model reduced.

Formally, let $L(\alpha \mid y)$ be the likelihood of the VAR model for data $y$ and let $g_{j}\left(\alpha \mid \gamma_{j}, M_{j}\right)$ be the prior induced by the DSGE model $M_{j}$ using parameters $\gamma_{j}$ on the VAR parameters $\alpha$.The marginal likelihood is $h_{j}\left(y \mid \gamma_{j}, M_{j}\right)=\int L(\alpha \mid y) g_{j}\left(\alpha \mid \gamma_{j}, M_{j}\right) d \alpha$, which, for given $y$, is a function of $M_{j}$. Since $L(\alpha \mid y)$ is fixed, $h_{j}\left(y \mid \gamma_{j}, M_{j}\right)$ reflects the plausibility of $g_{j}\left(\alpha \mid \gamma_{j}, M_{j}\right)$ in the data. Thus, if $g_{1}$ and $g_{2}$ are two DSGE-based priors and $h_{1}\left(y \mid \gamma_{1}, M_{1}\right)>h_{2}\left(y \mid \gamma_{2}, M_{2}\right)$, there is better support for in the data for $g_{1}$.

Thus, for a given data set, a researcher comparing the marginal likelihood produced by adding data from the exogenous and the endogenous specifications should detect whether the observable sample is more likely to be generated by one of the two models. We prefer to use the DSGE-VAR device rather 
than comparing the marginal likelihood of different models directly because small samples may led to distortions in marginal likelihood comparisons, distortions that will be reduced in our DSGE-VAR setup.

\begin{tabular}{|c|c|c|c|c|c|c|}
\hline & \multicolumn{3}{|c|}{$T_{1}=150$} & \multicolumn{3}{c|}{$T_{1}=750$} \\
\hline DGP & Model B & Model C & Model D & Model B & Model C & Model D \\
\hline Simulated from B & 1.00 & 0.00 & 0.00 & 0.99 & 0.00 & 0.00 \\
Simulated from C & 0.01 & 0.99 & 0.00 & 0.00 & 0.98 & 0.00 \\
Simulated from D & 0.00 & 0.00 & 1.00 & 0.00 & 0.00 & 0.99 \\
\hline
\end{tabular}

Table 2: Probability that Bayes factor exceeds 3.0 in a sample of 1,000 experiments. Marginal likelihoods are obtained using $\mathrm{T}=150$ data points produced by the models listed in the first row and $T_{1}$ simulated data from the model listed in the first column. When rows do not sum to one, the Bayes factor is inconclusive (below 3.0).

Table 2 reports results using this technology in the RBC example. The sample size is $T=150$ and Bayes factors computed when $T_{1}=150,750$ simulated data from the DSGE listed in the first row are added to the actual data and 1,000 experiments are run. The statistic is powerful since marginal likelihood differences are quite large, even when $T_{1}=150$.

\subsection{Some practical suggestions}

Given that, in practice, we do not know if a model is misspecified or not, we suggest users the following checklist as a way to approach the diagnostic problem:

i) Take a conventional model that has been used and tested in the literature and estimate its structural parameters, potentially allowing for time variations in the variance of the shocks.

ii) Run the time variation diagnostics and, if time variations are found to be present, check whether endogenous vs. exogenous variations are more appropriate.

When the model is of large scale, running regressions on all potential endogenous variables leads to overparameterization and muticollinearity. Thus, it is important to select the relevant variables to make the test powerful. We recommend users to employ the states of the model, as they determine the endogenous variables. Similarly, when performing the exogenous vs. endogenous check, having the proper state variables for the endogenous specification is important to make the comparison fair. One way do this is to estimate a model with exogenous time variation, take the smoothed residuals and run auxiliary regressions of the smoothed residuals on potential determinants of time variations. To avoid overparameterization, we also suggest users to a-priori shrink the coefficients of the auxiliary diagnostic regressions toward zero. Rejection of the null of no time variations in this case provides stronger confidence that parameter variations are indeed present. 
When the diagnostics detect time variations, one needs to specify which parameter may be time varying for the next stage of the analysis. In theory, one could specify time variations in all the structural parameters of interest, but this may lead again to an overparametrized model, which is difficult to estimate. We suggest two approaches here: either introduce time variations in parameters which have been documented in the literature to be unstable or in parameters a researcher suspects variations to be present. Alternatively, one could look at the smoothed residuals of the time invariant model, equation by equation, and restrict time variations to the parameters appearing of the equations whose residuals show the largest evidence of serial correlation.

\section{Parameter identification}

Since forecast errors are used to construct the likelihood function via the Kalman filter, one should expect the misspecification present in the forecast errors to spread to the likelihood function. In this section we examine whether time invariant parameters can be identified from a potentially misspecified likelihood function. Canova and Sala (2009) have shown that standard DSGE models feature several population identification problems, intrinsic to the models and to the solution method employed. The issue we are concerned with here is whether parameters that could be identified if the correct likelihood is employed became poorly identified when the wrong likelihood is used. In other words, we ask whether identification problems in time invariant parameters may emerge as a byproduct of neglecting variations in other parameters. Magnusson and Mavroedis (2014) have shown that when GMM is used, time variations in certain parameters help the identification of time invariant parameters. Huang (2014) qualifies the result by showing that time variations in weakly identified parameters have no effect on the asymptotic distribution of strongly identified parameters.

Figures 2 and 3 plot the likelihood function of the $\mathrm{RBC}$ model in the risk aversion coefficient $\gamma$ and the share parameter $\eta$; and in the labor share $\alpha$ and the autoregressive parameter of the technology $\rho_{\zeta}$, when the forecast errors of the correct model (top row) and of the constant coefficient model (bottom row) are used to construct the likelihood function. The first column considers data generated by the model $\mathrm{B}$, the second and the third data generated by models $\mathrm{C}$ and $\mathrm{D}$.

While the likelihood curvature in the correct model is not large, it is easy to verify that the maximum occurs at $\gamma=2, \eta=2, \alpha=0.30, \rho_{\zeta}=0.9$ for all three specifications. When the decision rules of the constant coefficients model are used to construct the likelihood function and the true DGP is model B, the likelihood is flattened and the risk aversion coefficient $\gamma$ become very weakly identified. When the true model features endogenous time variations, distortions are larger. The likelihood function becomes locally convex in $\rho_{\zeta} ; \gamma$ and $\alpha$ become weakly identified, and the maximum in the $\rho_{\zeta}$ is shifted away from the true value. 
True RBC B - Estimated with RBC Brue RBC C - Estimated with RBC Grue RBC D - Estimated with RBC D
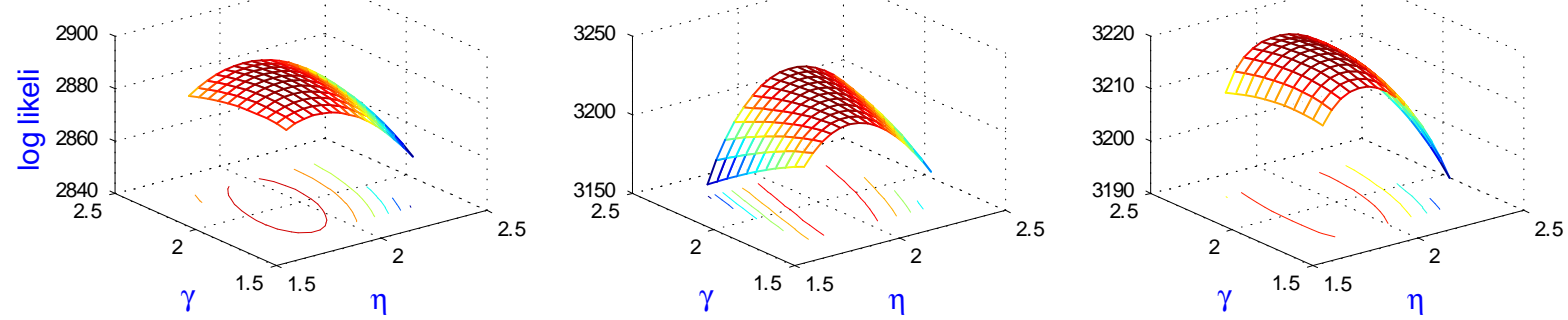

True RBC B - Estimated with RBC ATrue RBC C - Estimated with RBC Arue RBC D - Estimated with RBC A
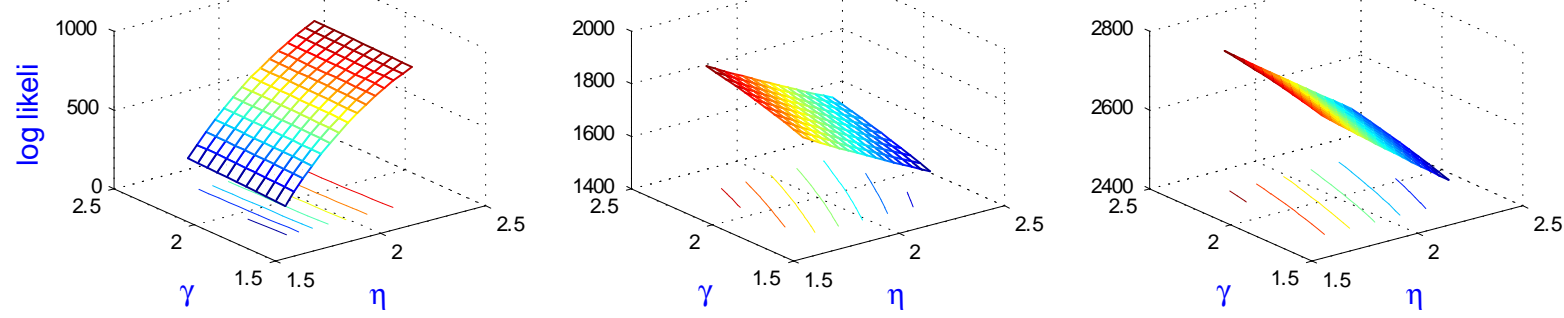

Figure 2: Likelihood surfaces

True RBC B - Estimated with RBC Brue RBC C - Estimated with RBC Crue RBC D - Estimated with RBC D
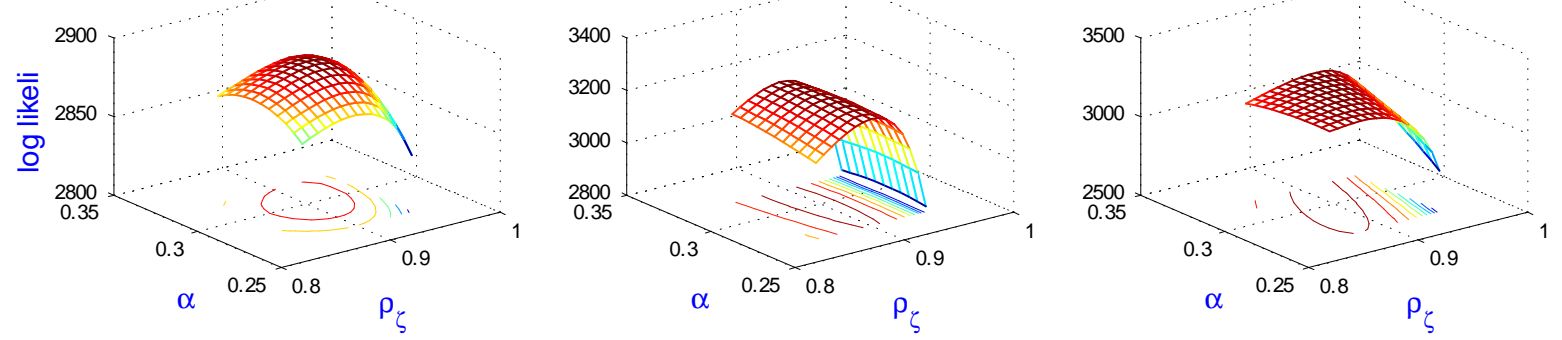

True RBC B - Estimated with RBC Arue RBC C - Estimated with RBC Arue RBC D - Estimated with RBC A
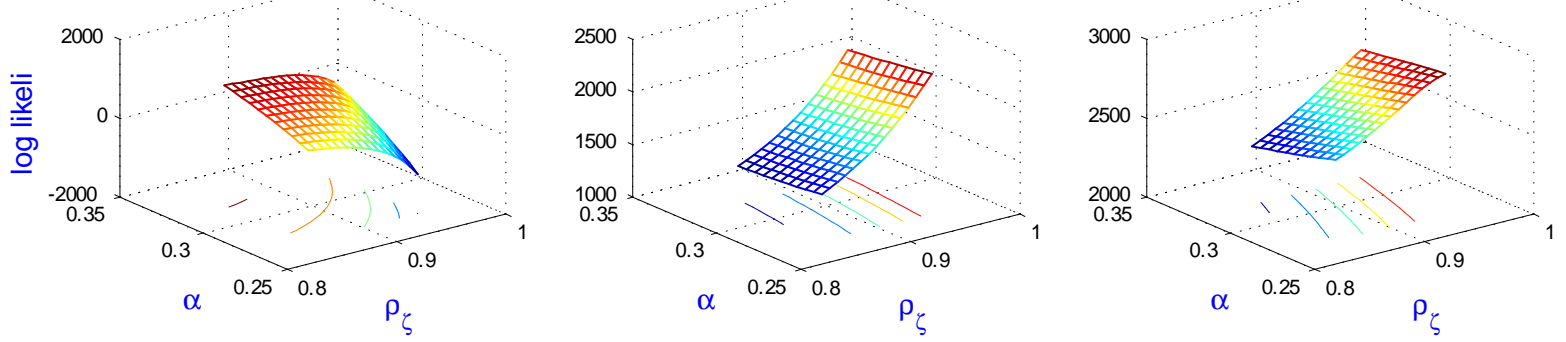

Figure 3: Likelihood surfaces 
These observations are confirmed by the Koop et al. (2013) statistic, see table 4. Koop et al. show that asymptotically the precision matrix grows at the rate $T$ for identified parameters and at rate less than $\mathrm{T}$ for underidentified parameters. Thus, the precision of the estimates, scaled by the sample size, converges to a constant for identified parameters and to zero for underidentified parameters. Furthermore, the magnitude of the constant measures identification strength: a large value indicates a strongly identified parameter; a small value a weakly identified one.

\begin{tabular}{|c|c|c|c|c|c|c|c|}
\hline Parameter & $\mathrm{T}=150$ & $\mathrm{~T}=300$ & $\mathrm{~T}=500$ & $\mathrm{~T}=750$ & $\mathrm{~T}=1000$ & $\mathrm{~T}=1500$ & $\mathrm{~T}=2500$ \\
\hline & \multicolumn{7}{|c|}{$\mathrm{DGP}$ Model B, Estimated model A } \\
\hline$\gamma$ & 15.9 & 17.8 & 17.2 & 18.8 & 18.4 & 19.3 & 17.9 \\
$\gamma$ & 28.5 & 45.7 & 108.4 & 81.4 & 93.6 & 104.2 & 90.17 \\
$\rho_{z}$ & $1.8 \mathrm{e}+4$ & $2.6 \mathrm{e}+4$ & $4.2 \mathrm{e}+4$ & $4.2 \mathrm{e}+4$ & $4.5 \mathrm{e}+4$ & $4.9 \mathrm{e}+4$ & $4.37 \mathrm{e}+4$ \\
$\rho_{g}$ & 209.2 & 655.5 & 2741 & 2190 & 2860 & 3417 & 2802 \\
$\delta$ & 927.3 & 973.8 & $1.7 \mathrm{e}+4$ & $1.7 \mathrm{e}+4$ & $2.4 \mathrm{e}+4$ & $2.3 \mathrm{e}+4$ & $2.5 \mathrm{e}+4$ \\
$\alpha$ & 140.2 & 156.2 & 264.2 & 215.5 & 239.1 & 252.1 & 229.3 \\
$A$ & 28.42 & 30.67 & 7.99 & 10.99 & 9.15 & 7.83 & 9.83 \\
\hline \multicolumn{7}{|c|}{ DGP Model C, Estimated model A } \\
\hline$\eta$ & 822 & 1033 & 743 & 785 & 759 & 746 & 752 \\
$\gamma$ & 2261 & 3147 & 2682 & 2809 & 2720 & 2579 & 2566 \\
$\rho_{z}$ & 3073 & 2673 & 2952 & 2909 & 2799 & 2806 & 2877 \\
$\rho_{g}$ & 1.74 & 2.23 & 2.44 & 2.96 & 3.17 & 2.82 & 2.90 \\
$\delta$ & $4.6 \mathrm{e}+5$ & $4.4 \mathrm{e}+5$ & $4.3 \mathrm{e}+5$ & $4.0 \mathrm{e}+5$ & $3.8 \mathrm{e}+5$ & $4.4 \mathrm{e}+5$ & $4.3 \mathrm{e}+5$ \\
$\alpha$ & $1.8 \mathrm{e}+4$ & $1.1 \mathrm{e}+4$ & $1.4 \mathrm{e}+4$ & $1.2 \mathrm{e}+4$ & $1.1 \mathrm{e}+4$ & $1.6 \mathrm{e}+4$ & $1.5 \mathrm{e}+4$ \\
$A$ & 351 & 493 & 441 & 505 & 500 & 449 & 444 \\
\hline \multicolumn{7}{|c|}{ DGP Model D, Estimated model A } \\
\hline$\eta$ & 550 & 575 & 592 & 610 & 545 & 542 & 494 \\
$\gamma$ & 3577 & 2442 & 2660 & 2870 & 2564 & 2711 & 2430 \\
$\rho_{z}$ & 1613 & 1243 & 1120 & 1162 & 1068 & 1189 & 1074 \\
$\rho_{g}$ & 1.22 & 1.28 & 1.44 & 1.53 & 1.60 & 1.62 & 1.67 \\
$\delta$ & $5.2 \mathrm{e}+5$ & $6.7 \mathrm{e}+5$ & $6.5 \mathrm{e}+5$ & $6.0 \mathrm{e}+5$ & $5.7 \mathrm{e}+5$ & $5.8 \mathrm{e}+5$ & $5.7 \mathrm{e}+5$ \\
$\alpha$ & $1.1 \mathrm{e}+4$ & $2.5 \mathrm{e}+4$ & $2.4 \mathrm{e}+4$ & $1.9 \mathrm{e}+4$ & $2.1 \mathrm{e}+4$ & $2.0 \mathrm{e}+4$ & $2.1 \mathrm{e}+4$ \\
$A$ & 488 & 276 & 340 & 382 & 349 & 395 & 334 \\
\hline \hline
\end{tabular}

Table 3: Koop, Pesaran, and Smith diagnostic. Reported are the diagonal elements of the precision matrix scaled by the sample size

When the DGP is model B and a fixed coefficients model is considered, all parameters are identified, even though $A$ and $\eta$ are only weakly identified. When the DGP are models $\mathrm{C}$ and $\mathrm{D}$, all parameters but $\rho_{g}$ seem identifiable. Interestingly, in models $\mathrm{C}$ and $\mathrm{D}, \rho_{g}$ is weakly identified, even when the correct likelihood is used. Thus, time variations in $\beta_{t}$ and $\delta_{t}$ do not help in the identification of $\rho_{g}$, in line with Huang (2014). 


\section{Structural estimation with a misspecified model}

To study the properties of likelihood -based estimates of a misspecified constant coefficients model, we conduct a Monte Carlo exercise. We generate 150 or 1,000 data points from versions $\mathrm{B}, \mathrm{C}, \mathrm{D}$ of the $\mathrm{RBC}$ model previously considered, estimate the structural parameters using the likelihood function constructed with the decision rules of the time invariant model $\mathrm{A}$, and repeat the exercise 150 times using different shock realizations. We also estimate the structural parameters using the likelihood constructed with the correct decision rules (i.e. model B rules if the data is generated with model $\mathrm{B}$, etc.) for benchmarking estimation distortions.

We consider two setups: one where parameter variations are small (2-5 percent of the variance of output is explained by shocks to the parameters; henceforth, DGP1) and one where parameter variations are substantial (around 20 percent of the variance of output is explained by shocks to the parameters; henceforth, DGP2). Table 4 has the results for DGP1: it reports the fixed parameters used to generate the data (column 1), the mean posterior estimate (across replications) obtained when the likelihood uses the correct decision rules (column 2), and the mean posterior estimate, the 5th and the 95th percentile of the distribution of estimates obtained when the likelihood function uses the decision rules of the time invariant model, when $\mathrm{T}=150$ (columns 3-5) and when $\mathrm{T}=1000$ (columns 6-8). Table A1 in the appendix has the results for DGP2. Figures A1 and A2 in the appendix plot the distributions of estimates for the two DGPs. The vertical line represents the true parameter value; solid black lines represent distributions obtained with the correct model; solid blue (red) lines represent the distributions obtained with the incorrect constant coefficient model when $\mathrm{T}=150(\mathrm{~T}=1000)$. When the model is correctly specified, the distribution of estimates should collapse around the true value. Thus, if the mean is away from the true parameter value and/or the spread of the distribution is large, likelihood -based methods have difficulties in recovering the constant parameters of the data generating process. Figure 4 presents the impulse responses for DGP1: the first two columns have the responses to technology shocks and government expenditure shocks in model $\mathrm{B}$, the next two the responses in model $\mathrm{C}$, and the last two the responses in model $\mathrm{D}$. In each box we report the response obtained using mean value of the correct distribution of estimates, and the 16th and 84th percentiles of the distribution of responses obtained using the estimated distribution of parameters produced by the time invariant model. Figure A3 in the appendix has the same information for DGP2. Table 5 presents the long run variance decomposition for DGP1 (table A2 has the information for DGP2) when $\mathrm{T}=150$ and the mean posterior estimate is used in the computations. In the first two columns we have the contribution of technology and government spending shocks in the correct model; the last two columns have the contribution when the constant coefficient model is used.

For the two time varying parameters, we set $d_{t}=\beta_{t+1} / \beta_{t}$, and assume that in model $\mathrm{B}, \Theta_{t+1}-\Theta \equiv\left(d_{t+1}\left(1-\rho_{\beta}\right) \beta, \delta_{t+1}-\left(1-\rho_{\delta}\right) \delta\right)^{\prime}=U_{t+1}$, where $\beta=0.99, \delta=$ 0.025 the components of $U_{t+1}=\left(u_{d, t+1}, u_{\delta, t+1}\right)^{\prime}$ are independent $\operatorname{AR}(1)$ process with 
persistence $\rho_{d}=0.9, \rho_{\delta}=0.8$, and standard deviations $\sigma_{d}=0.002, \sigma_{\delta}=0.07$. For models $\mathrm{C}$ and $\mathrm{D}$, the law of motion of the time varying parameters is $\Theta_{t+1}=\left[\Theta_{u}-\right.$ $\left.\left(\Theta_{u}-\Theta_{l}\right) e^{-\phi_{a}\left(K_{t}-K\right)}\right]+\left[\Theta_{u}-\left(\Theta_{u}-\Theta_{l}\right) e^{\phi_{b}\left(K_{t}-K\right)}\right]+U_{t+1}$, where $\Theta_{u}^{\prime}=(0.9999,0.03)$, $\phi_{a}^{\prime}=(0.03,0.2), \phi_{b}^{\prime}=(0.031,0.1), U_{t+1}$ is i.i.d. with $\Sigma_{u}=\operatorname{diag}(0.03,0.008)$.

\begin{tabular}{|c|c|c|c|c|c|c|c|}
\hline True value & $\begin{array}{c}\text { Estimated Correct } \\
\text { Mean } \\
\mathrm{T}=150\end{array}$ & $\begin{array}{r}\text { Est } \\
\text { Mean }\end{array}$ & $\begin{array}{l}\text { mated } \mathrm{Ti} \\
\text { percenti } \\
\mathrm{T}=150\end{array}$ & $\begin{array}{l}\text { avariant } \\
\text { percentile }\end{array}$ & $\begin{array}{r}\text { Est } \\
\text { Mean }\end{array}$ & $\begin{array}{l}\text { nated } \mathrm{T} \\
\text { percent } \\
\mathrm{T}=100\end{array}$ & $\begin{array}{l}\text { variant } \\
\text { percentile }\end{array}$ \\
\hline & & & DGP 1 & & & & \\
\hline$\eta=2.0$ & 2.00 & 2.03 & 1.47 & 2.88 & 2.32 & 1.55 & 3.37 \\
\hline$\gamma=2.0$ & 2.02 & 1.23 & -0.14 & 2.07 & 0.96 & -0.38 & 2.04 \\
\hline$\rho_{z}=0.98$ & 0.97 & 0.99 & 0.97 & 1.00 & 0.99 & 0.96 & 1.00 \\
\hline$\rho_{g}=0.5$ & 0.47 & 0.74 & 0.60 & 0.96 & 0.87 & 0.77 & 0.98 \\
\hline$\delta=0.025$ & 0.03 & 0.01 & 0.01 & 0.02 & 0.01 & 0.01 & 0.05 \\
\hline$\alpha=0.3$ & 0.30 & 0.19 & 0.11 & 0.28 & 0.23 & 0.15 & 0.40 \\
\hline$A=4.5$ & 4.55 & 2.79 & 1.33 & 4.12 & 2.68 & 1.23 & 4.06 \\
\hline & & & DGP 1 & & & & \\
\hline$\eta=2.0$ & 2.00 & 2.42 & 1.63 & 3.85 & 2.85 & 1.73 & 6.14 \\
\hline$\gamma=2.0$ & 2.00 & 0.64 & -0.26 & 1.77 & 0.60 & -0.50 & 1.79 \\
\hline$\rho_{z}=0.98$ & 0.98 & 0.99 & 0.97 & 1.00 & 0.97 & 0.85 & 1.00 \\
\hline$\rho_{g}=0.5$ & 0.48 & 0.43 & -0.10 & 0.96 & 0.65 & 0.27 & 0.98 \\
\hline$\delta=0.025$ & 0.03 & 0.01 & 0.01 & 0.02 & 0.02 & 0.01 & 0.09 \\
\hline$\alpha=0.3$ & 0.30 & 0.22 & 0.13 & 0.34 & 0.29 & 0.18 & 0.47 \\
\hline$A=4.5$ & 4.49 & 2.14 & 1.18 & 3.47 & 2.37 & 1.18 & 3.66 \\
\hline & & & $\overline{\mathrm{DGP} \mathrm{N}}$ & & & & \\
\hline$\eta=2.0$ & 2.00 & 2.58 & 1.69 & 3.34 & 2.40 & 1.74 & 3.26 \\
\hline$\gamma=2.0$ & 2.01 & 0.29 & -0.28 & 1.54 & 1.09 & -0.30 & 1.99 \\
\hline$\rho_{z}=0.97$ & 0.96 & 0.99 & 0.94 & 1.00 & 0.96 & 0.91 & 1.00 \\
\hline$\rho_{g}=0.5$ & 0.48 & 0.51 & -0.26 & 0.96 & 0.66 & 0.39 & 0.98 \\
\hline$\delta=0.025$ & 0.02 & 0.01 & 0.01 & 0.03 & 0.01 & 0.01 & 0.02 \\
\hline$\alpha=0.3$ & 0.30 & 0.22 & 0.14 & 0.35 & 0.22 & 0.15 & 0.30 \\
\hline$A=4.5$ & 4.52 & 2.32 & 1.42 & 3.68 & 3.45 & 1.37 & 4.51 \\
\hline
\end{tabular}

Table 4: Distributions of estimates, DGP1.

A few features of the results are worth discussing. First, when the correct model is employed, estimation is successful even when $\mathrm{T}=150$, regardless of the DGP and of whether time variations are exogenous or endogenous. Thus, numerical distortions seem minor. Second, with DGP1, a number of distortions occur when a time invariant model is used in estimation. For example, when exogenous variations are present, the persistence of government spending shock is poorly estimated (mean persistence is about 50 percent larger than the true one), while estimates of $\delta, \alpha$ and $A$ are severely 
biased downward. The distortions are smaller when the time variations are endogenous (models C and D). Nevertheless, significant downward biases exist in the inverse of the Frisch elasticity $\gamma$, in $\delta$ and $\alpha$. Third, the performance of the time invariant model is roughly independent of whether the data features external or internal endogenous time variations and does not improve when the sample size increases.

When parameter variations explain a significant portion of output variability, all features become more striking. For example, when parameter variations are exogenous, estimating a time invariant model leads to an overestimation of the persistence of the structural shocks. In fact, the only way a time invariant model can accommodate the additional dynamics and variability present in the endogenous variables is by increasing the persistence of both shocks. In models $\mathrm{C}$ and $\mathrm{D}$ the distortions become considerably larger and, for example, the mean posterior estimate of inverse of the Frisch elasticity is now negative. In addition, the distribution of estimates is typically skewed and multimodal. Thus, neglected parameter variations are more detrimental when they account for a significant portion of the variability of the endogenous variables.
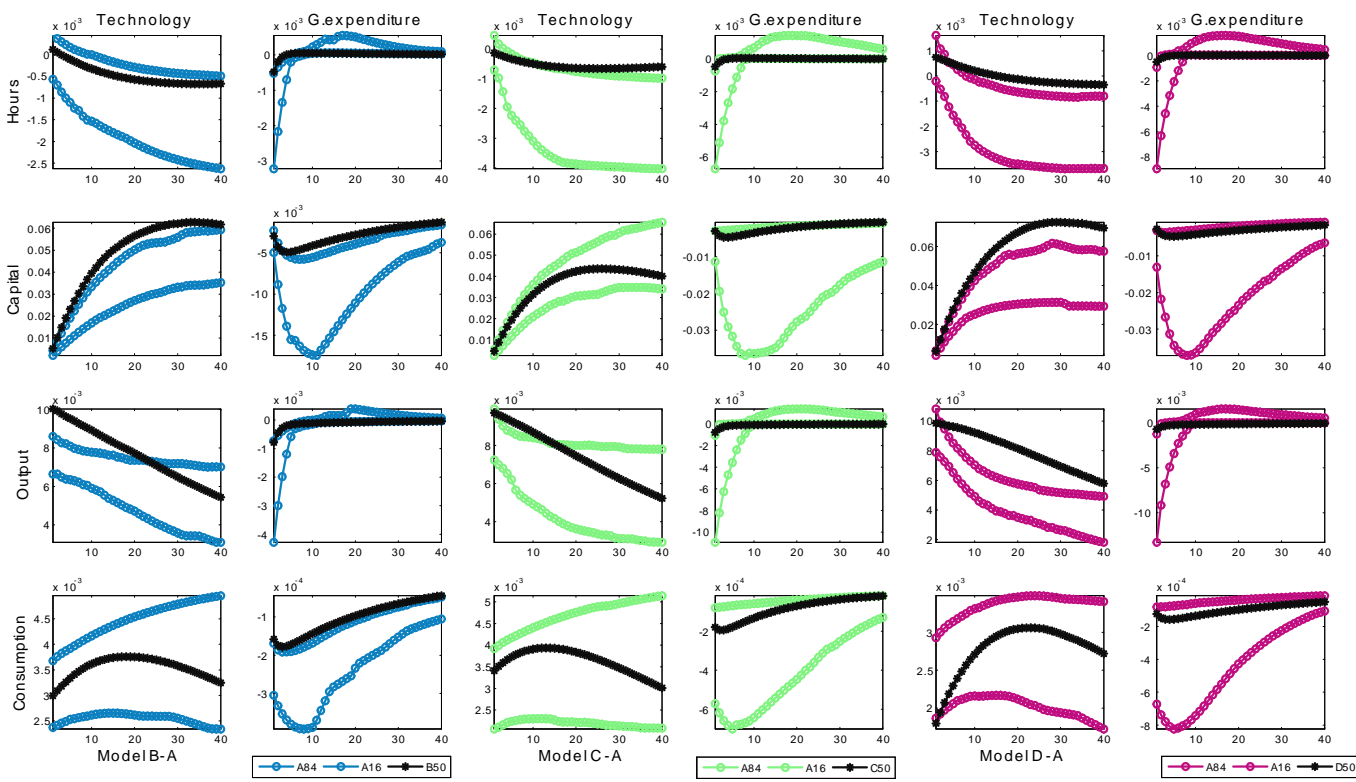

Figure 4: Impulse responses, DGP1

Impulse responses are in line with these conclusions. When parameter variations explain a small fraction of the variability of output, responses to technology shocks are off in terms of impact magnitude, in particular for output; and the response produced with estimates of the true model tend to be on the upper limit of the estimated 68 percent band produced with estimates of the incorrect model. Interestingly, output responses are those more poorly characterized and, consistent with previous findings, the misspecification is larger when the true model features exogenous time variations. 
The responses to government expenditure shocks obtained with a time invariant model are different from those obtained estimating the correct model in terms of magnitude, shape and persistence. Since the signal that government expenditure shocks produce is weak, it is not surprising that it is obscured by the presence of parameter variations.

The dynamic distortions obtained when parameter variations matter for the variance of output are generally larger. For example, the persistence of the responses to technology shocks is poorly estimated. While true responses tend to zero, the bands obtained estimating a time invariant model do not include zero even after 10 years.

\begin{tabular}{|l|c|c|c|c|}
\hline Variable & Technology & Government & Technology & Government \\
\hline & \multicolumn{2}{|c|}{ DGP: Model B } & \multicolumn{2}{|c|}{ Estimated: Time invariant } \\
\hline Y & 94.100 & 0.300 & 0.997 & 0.004 \\
$\mathrm{C}$ & 89.500 & 0.200 & 0.999 & 0.001 \\
$\mathrm{~N}$ & 60.200 & 0.500 & 0.986 & 0.014 \\
$\mathrm{~K}$ & 70.200 & 0.400 & 0.995 & 0.006 \\
\hline & \multicolumn{2}{|c|}{ DGP: Model C } & \multicolumn{2}{|c|}{ Estimated: Time invariant } \\
\hline $\mathrm{Y}$ & 97.200 & 0.300 & 0.988 & 0.016 \\
$\mathrm{C}$ & 88.100 & 0.300 & 0.999 & 0.001 \\
$\mathrm{~N}$ & 44.600 & 0.600 & 0.990 & 0.012 \\
$\mathrm{~K}$ & 84.400 & 0.200 & 0.990 & 0.014 \\
\hline & \multicolumn{2}{|c|}{ DGP: Model D } & Estimated: Time invariant \\
\hline $\mathrm{Y}$ & 98.000 & 0.100 & 0.993 & 0.015 \\
$\mathrm{C}$ & 92.200 & 0.200 & 0.998 & 0.003 \\
$\mathrm{~N}$ & 35.900 & 0.500 & 0.973 & 0.034 \\
$\mathrm{~K}$ & 96.600 & 0.300 & 0.992 & 0.012 \\
\hline
\end{tabular}

Table 5: Long run variance decomposition, DGP1.

What is the contribution of structural shocks to the variability of the endogenous variables when the forecast errors of the time invariant model are used to construct the likelihood function? One should expect the structural shocks of the time invariant model to be a contaminated version of the structural shocks of the time varying DGP for two reasons. First, the wrong P matrix is used to compute forecast errors. Second, we are aggregating $\mathrm{m}$ (primitive and parameter) shocks into $n<m$ (structural) shocks, thus generating VARMA decision rules where the $\mathrm{n}$ structural shocks are functions of the leads and lags of the original disturbances (see e.g. Canova and Paustian, 2011). Thus, even if the P matrix were correctly specified, distortions should occur, unless the shocks to the parameters are unimportant and feature low persistence.

When parameter variations explain a small portion of output, technology shocks in the time invariant model absorb the missing variability, regardless of the nature of parameter variations and the effect seems strong for hours worked. When parameter variations explain a larger portion of the variance of output, technology shocks still absorb a large amount of the missing variability but, in some cases, spending shocks 
also capture the missing variability see, e.g., the case of endogenous variations ${ }^{2}$

In sum, for the DGP we consider and the parameterization employed, estimating a constant parameter model when the DGP features time varying parameters leads to distortions, regardless of the sample size, of whether variations are exogenous or endogenous, and of whether parameter variations matter for output variability or not. The parameters mostly affected are those regulating the estimated persistence of the shocks and those controlling income and substitution effects.

\section{Structural dynamics and SVAR methods}

The previous section showed that if time variations are neglected, structural estimates are biased and structural responses distorted. Because of these problems, one may wonder whether less structural and computationally less demanding methods can be used if structural dynamics are all that matters to the investigator. Canova and Paustian (2011) showed that when the model misses features of the DGP, SVAR methods employing robust sign restrictions can be effective in capturing qualitative features of structural dynamics. Here we ask if SVARs are good also when parameter variations are neglected.

The exercise is as follows. Using the illustrative RBC model, we simulate data from the decision rules of models $\mathrm{B}, \mathrm{C}$, and $\mathrm{D}$ when parameter variations generate small output volatility (DGP1). We then estimate a VAR, compute residuals, and rotate them using an orthonormal matrix. We then keep the resulting impulse responses if (simultaneously) technology shocks generate a positive response of hours, capital, output, and consumption on impact and government expenditure shocks generate a negative response of hours, output, consumption and capital. These restrictions hold in the four model specifications we consider and are robust to variations of the (constant) structural parameters within a reasonable range. We repeat the exercise 150 times and collect the distribution of structural responses when the correct and the time invariant SVAR specifications are used. Figure 5 plots the median response in the correct model (red line) and the 16th and 84th percentiles of the distribution of responses obtained with the time invariant model.

Overall, SVAR methods are competitive with structural methods when parameter variations are neglected. When the DGP is model $\mathrm{B}$, the sign and the shape of the responses are correctly captured. Although the responses to technology shocks obtained with the correct model are on the upper bound of the band obtained with the time invariant model and the responses to government spending shocks

\footnotetext{
${ }^{2}$ We have also performed a Monte Carlo exercise allowing the labor share to be time varying. Variations in the labor share have been documented in, e.g., Rios Rull and Santaeularia Llopis (2010), and there is evidence that they are strongly countercyclical. This is relevant for our exercise because all four optimatility conditions are affected by time variations, altering the strength of the income and substitution effect distortions. Indeed, we do find that distortions become quite large and it many cases it becomes difficult to estimate the time invariant model regardless of the DGP (results available on request).
} 
obtained with the correct model tend to be on the lower bound of the bands obtained, no major distortions occur. The performance with the other two DGPs is similar. With model $\mathrm{C}$, it is the magnitude of the response of consumption that is mainly misrepresented, while with model $\mathrm{D}$ it is primarily the persistence of certain responses that is underestimated.

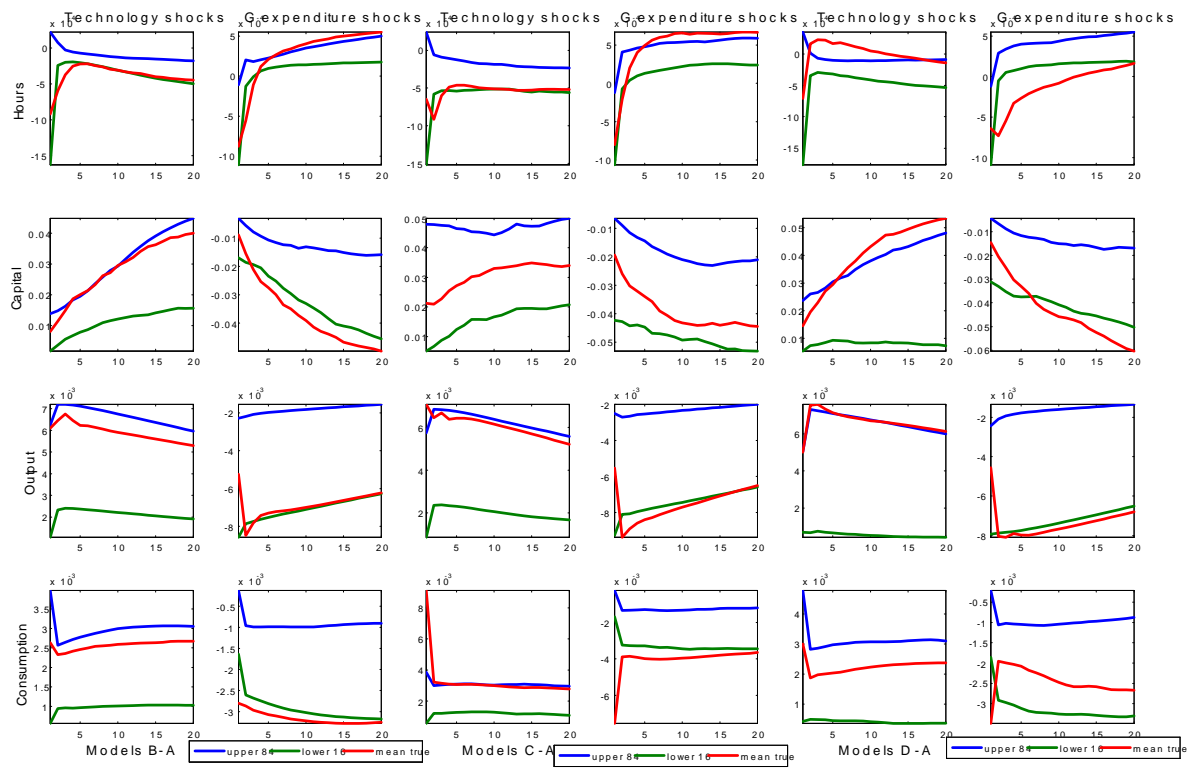

Figure 5: Impulse responses, DGP1, SVAR models.

Recall that there are two sources of misspecification in time invariant models: the $\mathrm{P}$ matrix is generally incorrect; aggregation problems are present. Our analysis indicates that, with the DGP we use, i) distortion in the $\mathrm{P}$ matrix are small; ii) the $\mathrm{Q}$ matrix is not very strongly affected by time variations; iii) shock misaggregation is minor. Because parameter shocks are i.i.d., timing distortions are also small.

\section{Time varying financial frictions?}

We apply the technology we developed to the unconventional monetary policy model of Gertler and Karadi's (GK) (2010). Our contribution is three fold. We provide likelihood estimates of the parameters specific to the model (the fraction of capital that can be diverted by banks $\lambda$, the proportional transfer to entering bankers $\omega$, and the survival probability of bankers $\theta$ ), which the authors have informally calibrated to match a steady state spread, a steady state leverage, and a notional length of bank activity; we use the diagnostics we developed to gauge the extent of parameter variations; we estimate a model with time variations in $\lambda$ and compare its fit with the 
fit of the time invariant model augmented with an extra shock; and examine responses to capital quality shocks in the fixed coefficient and the time varying coefficient models.

The equations of the GK model are summarized in appendix C. We use U.S. data from 1985Q2 to 2014Q3 on the growth rate of output, growth rate of consumption, growth rate of leverage, and growth intermediary demand for assets (credit) and the spread. The spread is measured by the difference between BAA 10-year corporate bond yields and a 10-year treasury constant maturity, and it is from the FRED, as are real personal consumption expenditures and GDP data. Leverage is from Haver and measures Tier 1 (core) capital as a percent of average total assets. Credit is measured as total loans (from Haver), scaled by size of US population. While the data transformation is sufficient to eliminate volatility variations, the credit and the spread variable display a significant structural break in the last few years of the sample. Thus, we present estimates obtained in the full sample and in the sample ending at 2007:4.

\begin{tabular}{|l|ccccc|c|}
\hline Equation & \multicolumn{5}{|c|}{ T-stat } & F-stat \\
\hline & $Y_{t-1}$ & $C_{t-1}$ & Credit \\
$t-1$ & Leverage $_{t-1}$ & Spread $_{t-1}$ & \\
\hline Y & \multicolumn{5}{|c|}{ Sample } & $1985: 3-2014: 3$ \\
C & 0.84 & 2.61 & 0.24 & 0.52 & 10.00 & 4.39 \\
Credit & -0.85 & 1.11 & 0.85 & -0.65 & 0.33 & 1.26 \\
Leverage & 1.06 & 2.61 & 1.65 & -0.58 & 8.49 & 7.11 \\
Spread & $-1.11-2.50$ & -1.63 & 0.63 & -8.25 & 7.04 \\
\hline & -1.26 & -3.06 & -1.10 & 0.81 & -8.46 & 8.16 \\
\hline Y & -1.79 & 3.87 & -2.23 & -0.38 & 6.86 & 4.23 \\
C & -1.37 & 1.19 & -0.26 & 0.38 & 1.40 & 0.81 \\
Credit & -1.18 & 3.53 & -0.69 & -0.08 & 7.02 & 3.60 \\
Leverage & $-1.06-3.46$ & 0.75 & 0.09 & -6.80 & 3.72 \\
Spread & 1.16 & -3.84 & -1.03 & 0.17 & -6.86 & 4.29 \\
\hline
\end{tabular}

Table 6: Regression diganostic for time variation. The left-hand side of the regression is the forecast in the equation listed in the first column; the right-hand side the variables listed in the second to the fifth column. Critical levels of the F-stat $(5,112)=2.56$ and $\mathrm{F}(5,85)=2.90$.

Using a flat prior, the posterior mode estimates for the full sample are $\lambda=0.245$, $\theta=0.464, \omega=0.012$; the standard errors are tight $(0.0182,0.0098,0.0008)$, making the estimates highly significant. For the shorter 1985-2007 sample the modal estimates are $\lambda=0.178, \theta=0.399, \omega=0.008$, and the standard errors are 0.0127, 0.0129, 0.0006 . Thus, while estimates of the three crucial parameters are altered when data for the last financial crisis is used, differences are small a-posteriori. For comparison, GK calibrated these three parameters to $\lambda=0.318, \theta=0.972, \omega=0.002$. In the GK model $\lambda$ regulates private leverage: our estimate implies a higher steady state leverage than the one implied by the authors (our estimate for the full sample is 3.32 , GK is 
1.38), which is closer to the leverage found in the U.S. in corporate and non-corporate business sectors over the sample. Our estimates also suggest that bankers' survival probability is lower than the one assumed by GK (about 10 years).

With the parameter estimates obtained in the full sample, we perform our diagnostics. Table 6 indicates that the forecast errors of all equations except consumption are predictable and, typically, lagged consumption and lagged spread matter. The mean value of the Euler wedge is 0.02 with a standard error of 0.03 ; but both lagged consumption and lagged investment to output ratios significantly explain its movements (coefficients are respectively -0.10 and 0.72 , with standard errors of 0.01 and 0.13 ). When we run our diagnostics on the shorter sample, we reach the same conclusions: all forecast errors but the one of the consumption equation are predictable, and lagged consumption and lagged spread matter; lagged consumption and lagged investment to output ratios predict the wedge (coefficients are respectively -0.13 and 0.96 , with standard errors of 0.02 and 0.23 ). Thus, the time variations we detect are not due to the crisis and to the potential break it generates.

\begin{tabular}{|l|cc|cc|cc|cc|}
\hline Parameter & Time Invariant & Time Invariant & \multicolumn{2}{|c|}{ Exogenous TVC } & \multicolumn{2}{|c|}{ Endogenous TVC } \\
\hline & & & \multicolumn{2}{|c|}{6 shocks } & & & Function of net worth \\
\hline $\mathrm{h}$ & 0.43 & $(0.006)$ & 0.11 & $(0.02)$ & 0.19 & $(0.03)$ & 0.09 & $(0.02)$ \\
$\lambda$ & 0.24 & $(0.01)$ & 0.97 & $(0.01)$ & 0.37 & $(0.03)$ & 0.55 & $(0.03)$ \\
$\omega$ & 0.01 & $(0.008)$ & 0.02 & $(0.001)$ & 0.02 & $(0.002)$ & 0.11 & $(0.008)$ \\
$\theta$ & 0.46 & $(0.009)$ & 0.80 & $(0.01)$ & 0.54 & $(0.01)$ & 0.52 & $(0.02)$ \\
\hline$\rho_{\lambda}$ & & & & & 0.99 & $(0.004)$ & & \\
$\sigma_{\lambda}$ & & & & & 0.02 & $(0.002)$ & 0.03 & $(0.003)$ \\
$\lambda_{u}$ & & & & & & & 0.98 & $(0.008)$ \\
$\phi_{1}$ & & & & & & & 0.02 & $(0.007)$ \\
$\phi_{2}$ & & & & & & & 0.15 & $(0.009)$ \\
\hline Log ML & -167.97 & 1098.32 & 1546.18 & & 1628.69 & \\
\hline
\end{tabular}

Table 7: Parameter estimates, Gertler and Karadi model, standard errors in parenthesis.

Armed with this preliminary evidence, we estimate the model allowing $\lambda$ to be time varying. Since $\lambda$ regulates leverage and drives movements in the credit and spread equations, whose smoothed residuals seem to be the most affected by serial correlation, we opt to make this parameter time varying. We specify

$$
\begin{gathered}
\lambda_{t}=\left(1-\rho_{\lambda}\right) \lambda+\rho_{\lambda} \lambda_{t-1}+e_{t, \lambda} \quad \text { Exogenous variations } \\
\lambda_{t}=\left(2 * \lambda_{u}-\left(\lambda_{u}-\frac{\lambda}{2}\right) *\left(\exp \left(-\phi_{1} *\left(X_{t-1}-X^{s}\right)\right)+\exp \left(\phi_{2} *\left(X_{t-1}-X^{s}\right)\right)\right)+e_{t, \lambda}\right. \\
\text { Endogenous variations }
\end{gathered}
$$

where $X$ is net bank wealth. We chose bank net wealth as the relevant state in the endogenous specification because of its importance for the spread and the credit variable. Table 7 reports estimates of selected parameters. 
In the model with exogenously varying parameters, variations in $\lambda_{t}$ very persistent. Furthermore, estimates of $(\lambda, \omega, \theta)$ are now larger making steady state leverage drop to about 2.9 and the lifetime of bankers to increase. With the endogenous specification, estimates of $\lambda$ and $\omega$ further increase, making steady state leverage fall to 1.9, but bankers' survival probability is roughly unchanged. The data seem to require a very strong asymmetric specification for time variations $\left(\phi_{1}<\phi_{2}\right)$, implying a strong negative relationship between the fraction of funds that bankers can divert and their net worth. Finally, note that, in term of marginal likelihood, the endogenous specification is superior to both the exogenous one and the fixed coefficient specification augmented by a shock to bankers' net worth accumulation equation.

To investigate how inference differs in the three estimated models, we plot in figure 7 the responses of output, inflation, investment, net worth, leverage, and the spread to a one percent capital quality shock. The constant coefficient specification closely replicates the dynamics presented by GK (see their figure 3). There is a persistent decline in output and a temporary but strong decline in inflation. Investment temporarily falls but it then increases because capital is below its steady state. Bankers' net worth falls and there is a sharp increase in the spread.
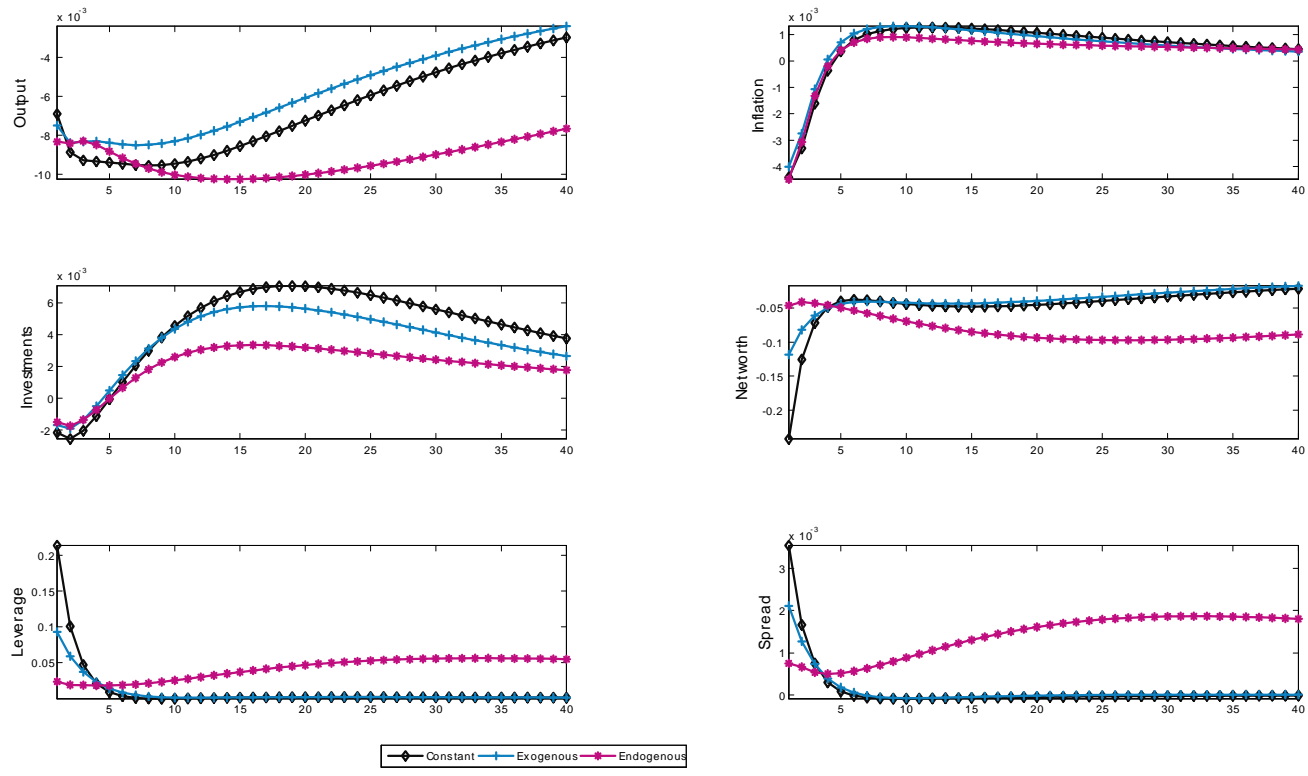

Figure 6: Dynamics in response to a capital quality shock

When we allow $\lambda$ to be exogenously varying, responses are qualitatively similar. Quantitatively, output falls more on impact but less in the short run; net worth falls less and the spread increases less in the short run. Thus, making $\lambda$ exogenously time varying, reduces the model's ability to capture recessionary effects on impact. 
When variations are endogenous, the model possesses an additional mechanism of propagation of shocks since lower net worth implies higher share of funds diverted by banks and generally stronger accelerator dynamics. Since the dynamic responses of net worth are highly persistent, the spread persistently increases making investment increase less and output fall more and more persistently relative to the other two cases. Thus, neglecting endogenous $\lambda$ variations could impair our ability to correctly measure the effects of capital quality shocks.

While the economic interpretation of the relationship between $\lambda$ and net worth is beyond the scope of the paper, attempts to endogenize crucial parameters in the Gertler and Karadi model exist (see e.g. Bi, Leeper and Leith, 2014; Ferrante, 2014). Their work could provide the microfundations for the evidence we uncover.

\section{Conclusions}

This paper is interested in i) characterizing the decision rules of a DSGE when parameter variations are exogenous or endogenous, and in the latter case, when agents internalize or not the effects their decisions may have on parameter variations; ii) providing diagnostics to detect misspecification driven by parameter variations; and iii) studying the consequences of using time invariant models when the parameters are time varying in terms of identification, estimation, and inference

We show that if parameter variations are purely exogenous, the contemporaneous impact and the dynamics induced by structural shocks are the same as in a model with no parameter variations. However, if parameter variations are endogenous, the structural dynamics may be different and the extent of the difference depends on the detail of the model. We provide diagnostics to detect the misspecification due to neglected parameter variations and describe a marginal likelihood diagnostics to recognize whether exogenous or endogenous time variations should be used.

We highlight that certain parameter identification problems noted in the literature may be the result of misspecification due to neglected time variations. Our Monte Carlo study indicates that parameter and impulse response distortions may be large even for modest time variations in the parameters. It also shows that, when parameter variations are neglected, SVAR methods are competitive with more structural likelihood-based methods, as far as the responses to structural disturbances are concerned.

In the context of the Gertler and Karadi (2010) model, we show that the parameter regulating the amount of moral hazard is likely to be time varying. When we allow variations to be linked to the amount of net worth bankers have, the fit of the model dramatically improves, primarily because there is an additional propagation channel that makes spread and thus output responses stronger and more persistent.

Overall, our analysis provide researchers with a new set of tools to help them to assess the quality of their models and respecify certain problematic features. 


\section{References}

Andreasen, M. (2012). On the effects of rare disasters and uncertainty shocks for risk premia in non-linear DSGE models. Review of Economic Dynamics, 15, 293-316.

Ascari, G. and A. Sbordone (2014). The macroeconomics of Trend Inflation. Journal of Economic Literature, 52, 679-739.

Bi, X. Leeper, E, C. Leith (2014). Financial Intermediation and Government debt default, University of Indiana, manuscript.

Brinca, P., Holter, H., P. Krusell, and L. Malafry (2014). Fiscal multipliers in the 21st century. Forthcoming,Journal of Monetary Economics.

Canova, F. (2009). What Explains the Great Moderation in the U.S.?: A Structural Analysis. Journal of the European Economic Association, 7, 697-721.

Canova, F. and L. Sala (2009). Back to square one: Identification issues in DSGE models. Journal of Monetary Economics, 56, 431-449.

Canova, F. and M. Paustian (2011). Business cycle measurement with some theory. Journal of Monetary Economics, 48, 345-361.

Chang, Y., Kim, S. and F. Schorfheide (2013). Labor market heterogeneity and the policy-(in)variance of DSGE model parameters'. Journal of the European Economic Association, 11,193-220.

Chari, V.V., Kehoe, P. and Mc Grattan, E. (2007). Business Cycle Accounting. Econometrica, 75, 781-836.

Cogley, T. and T. Yagihashi, (2010). Are DSGE approximating models invariant to policy shifts? The B.E. Journal of Macroeconomics (Contributions), Article 27.

Davig, T. and E. Leeper (2006). Endogenous monetary policy regime changes. NBER International Seminar in Macroeconomics, 345-391. National Bureau of Economic Research.

Del Negro, M. and F. Schorfheide (2004). Prior from general equilibrium models for VARs. International Economic Review, 45, 643-673.

Dueker, M. Fisher, A. and Dittman, R. (2006). Stochastic Capital Depreciation and the Co-movements of Hours and Productivity. Berkeley Journals: Topics in Macroeconomics, vol 6,3, article 6 .

Fernandez Villaverde, J. and J. Rubio Ramirez (2004). Comparing dynamic equilibrium economies to the data. Journal of Econometrics, 123, 153-187.

Fernandez Villaverde, J. and J. Rubio Ramirez (2007). How structural are structural parameters. NBER Macroeconomics Annual, 22, 83-132.

Ferrante, F. (2014) Endogenous loan quality, Federal Reserve Board, manuscript.

Galvao, A. , Giraitis, L., Kapetanios, G., and K. Petrova (2014). Local Bayesian estimation and forecasting with a TVP model, manuscript

Gertler, M. and Karadi, P. (2010). A Model of unconventional monetary policy. Journal of Monetary Economics, 58, 17-34.

Gourio, F. (2012). Disaster Risk and Business Cycles. American Economic Review, $102,2734-2766$. 
Huang, N. (2014). Weak Inference for DSGE models with time varying parameters. Boston College, manuscript.

Hurwicz, L. (1962). On the structural form of interdependent systems, in E. Nagel, P. Suppes, A. Tarski (eds.) Logic, Methodology and Philosophy of science: proceedings of a 1960 international congress. Stanford University Press.

Karabarbounis, L. and B. Neiman (2014). Capital depreciation and labor shares around the world: measurement and implications, manuscript.

Koop, G., Pesaran, H., and R. Smith (2013). On the identification of Bayesian DSGE models. Journal of Business and Economic Statistics, 31, 300-314

Kulish, M. and A. Pagan (2014) Estimation and Solution of models with expectation and structural changes, Dynare working paper 34 .

Inohue, A., Kuo, C.H. and B. Rossi, (2015) Identifying sources of model misspecification, UPF manuscript.

Ireland, P. (2004). Technology shocks in a New Keynesian model. Review of Economics and Statistics, 83, 926-933.

Ireland, P. (2007). Changes in the Federal Reserve Inflation target: Causes and Consequences. Journal of Money Credit and Banking, 39, 1851-1882.

Liu, Z., Waggoner, D. and T. Zha (2011). Sources of Macroeconomic fluctuations: A regime switching DSGE approach. Quantitative Economics, 2, 251-301.

Magnusson, L. and S. Mavroeidis (2014). Identification using stability restrictions. Econometrica 82, 1799-1851.

Meier S. and C.Sprengler (2015). Temporal Stability of Time preferences. Review of Economics and Statistics, 97(2), 273-286.

Mutschler, W. (2014) Identification of DSGE Models - the effect of higher order Approximation and Pruning, University of Munster, manuscript.

Rios Rull J. V. and R. SantaEularia-Llopis, (2010). Redistributive shocks and productivity shocks. Journal of Monetary Economics,37, 931-948.

Schmitt Grohe, S. and M. Uribe (2003). Closing small open economy models. Journal of International Economics, 62, 161-185.

Schmitt Grohe, S. and M. Uribe (2004). Solving Dynamic General Equilibrium Models using second order approximation to the policy function. Journal of Economic Dynamics and Control, 28, 755-775.

Seoane, H. (2014) Parameter Drifts, Misspecification and the Real Exchange Rate in Emerging Countries, manuscript, Universidad Carlos III Madrid.

Sims, C. (2001) Comment on T. Cogley and T. Sargent, Evolving US postwar dynamics, NBER macroeconomic Annual 16.

Stock, J. and Watson, M. (1996). Evidence of Structural Instability in Macroeconomic Relationships. Journal of Business and Economic Statistics,14, 11-30.

Vavra, J. (2014). Time varying Phillips curve, NBER working paper 19790. 
Appendix A: Additional Monte Carlo figures and tables
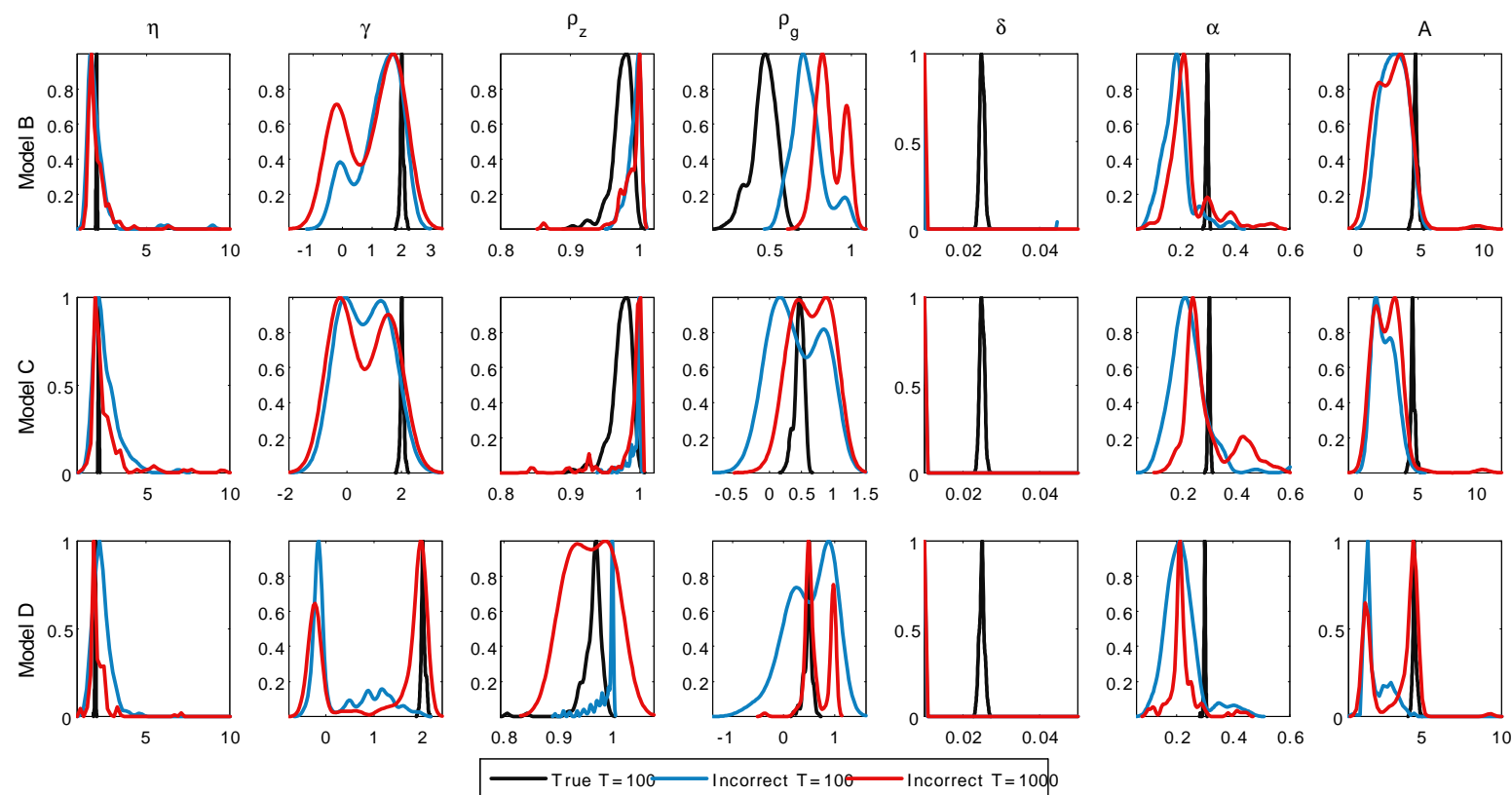

Figure A1: Density of estimates; DGP1 (parameter variations explain 2-5 percent of output variance). 


\begin{tabular}{|c|c|c|c|c|c|c|c|}
\hline \multirow[t]{2}{*}{ True } & $\begin{array}{c}\text { Estimated Correct } \\
\text { Mean }\end{array}$ & \multicolumn{3}{|c|}{$\begin{array}{c}\text { Estimated Time invariant } \\
\text { Mean } 5 \text { percentile } 95 \text { percentile }\end{array}$} & \multicolumn{3}{|c|}{$\begin{array}{c}\text { Estimated Time invariant } \\
\text { Mean } 5 \text { percentile } 95 \text { percentile }\end{array}$} \\
\hline & $\mathrm{T}=150$ & \multicolumn{3}{|c|}{$\mathrm{T}=150$} & \multicolumn{3}{|c|}{$\mathrm{T}=1000$} \\
\hline \multicolumn{8}{|c|}{ DGP Model B } \\
\hline$\eta=2.0$ & 2.00 & 2.29 & 1.53 & 3.87 & 2.45 & 1.61 & 3.09 \\
\hline$\gamma=2.0$ & 2.01 & 1.11 & -0.33 & 2.06 & 0.25 & -0.27 & 1.95 \\
\hline$\rho_{z}=0.9$ & 0.94 & 0.99 & 0.96 & 1.00 & 0.99 & 0.97 & 1.00 \\
\hline$\rho_{g}=0.5$ & 0.47 & 0.76 & 0.62 & 0.96 & 0.91 & 0.79 & 0.98 \\
\hline$\delta=0.025$ & 0.03 & 0.01 & 0.01 & 0.03 & 0.01 & 0.01 & 0.01 \\
\hline$\alpha=0.3$ & 0.30 & 0.19 & 0.11 & 0.41 & 0.21 & 0.10 & 0.34 \\
\hline$A=4.5$ & 4.53 & 2.73 & 1.33 & 4.14 & 1.80 & 1.14 & 4.16 \\
\hline \multicolumn{8}{|c|}{ DGP Model C } \\
\hline$\eta=2.0$ & 2.00 & 3.40 & 1.56 & 7.51 & 5.19 & 1.77 & 22.90 \\
\hline$\gamma=2.0$ & 2.00 & -0.08 & -0.32 & 0.73 & -0.19 & -0.35 & 0.35 \\
\hline$\rho_{z}=0.9$ & 0.88 & 0.99 & 0.93 & 1.00 & 0.99 & 0.90 & 1.00 \\
\hline$\rho_{g}=0.5$ & 0.48 & 0.56 & 0.08 & 0.97 & 0.91 & 0.59 & 0.98 \\
\hline$\delta=0.025$ & 0.02 & 0.02 & 0.01 & 0.07 & 0.02 & 0.01 & 0.07 \\
\hline$\alpha=0.3$ & 0.30 & 0.26 & 0.15 & 0.34 & 0.26 & 0.19 & 0.35 \\
\hline$A=4.5$ & 4.50 & 1.71 & 1.25 & 2.77 & 2.27 & 1.24 & 8.17 \\
\hline \multicolumn{8}{|c|}{ DGP Model D } \\
\hline$\eta=2.0$ & 2.00 & 3.05 & 1.68 & 4.59 & 2.40 & 1.98 & 4.81 \\
\hline$\gamma=2.0$ & 2.00 & -0.06 & -0.28 & 0.54 & 1.63 & -0.27 & 1.98 \\
\hline$\rho_{z}=0.9$ & 0.88 & 0.98 & 0.90 & 1.00 & 0.92 & 0.91 & 1.00 \\
\hline$\rho_{g}=0.5$ & 0.47 & 0.42 & -0.46 & 0.96 & 0.50 & 0.32 & 0.97 \\
\hline$\delta=0.025$ & 0.02 & 0.01 & 0.01 & 0.03 & 0.01 & 0.01 & 0.01 \\
\hline$\alpha=0.3$ & 0.30 & 0.23 & 0.15 & 0.32 & 0.21 & 0.13 & 0.27 \\
\hline$A=4.5$ & 4.49 & 1.91 & 1.45 & 3.57 & 4.10 & 1.65 & 4.51 \\
\hline
\end{tabular}

Table A1: Distributions of estimates, DGP2 (parameter variations explain 20 percent of output variance). 

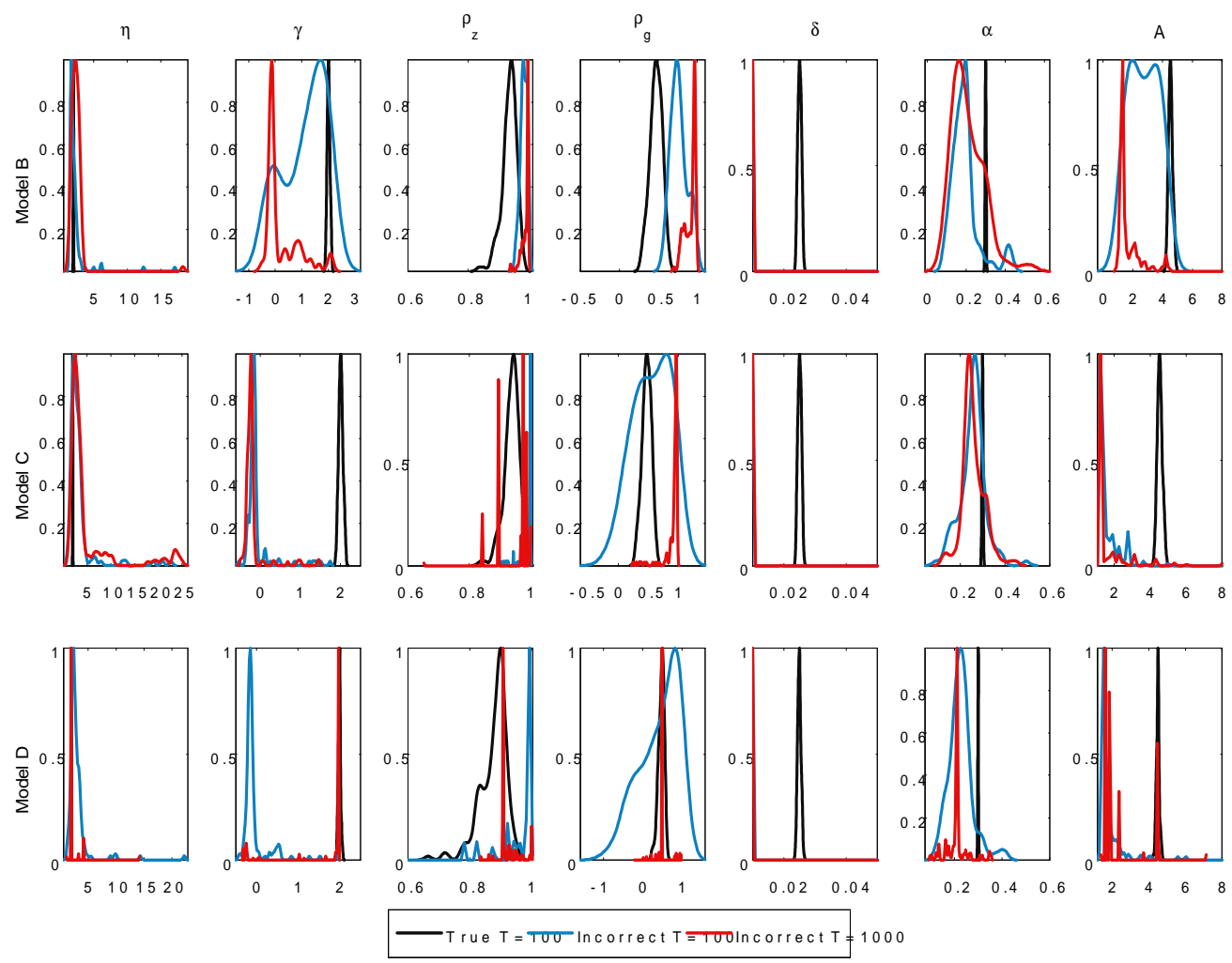

Figure A2: Density of estimates, DGP2 (parameter variations explain 20 percent of output variance) 

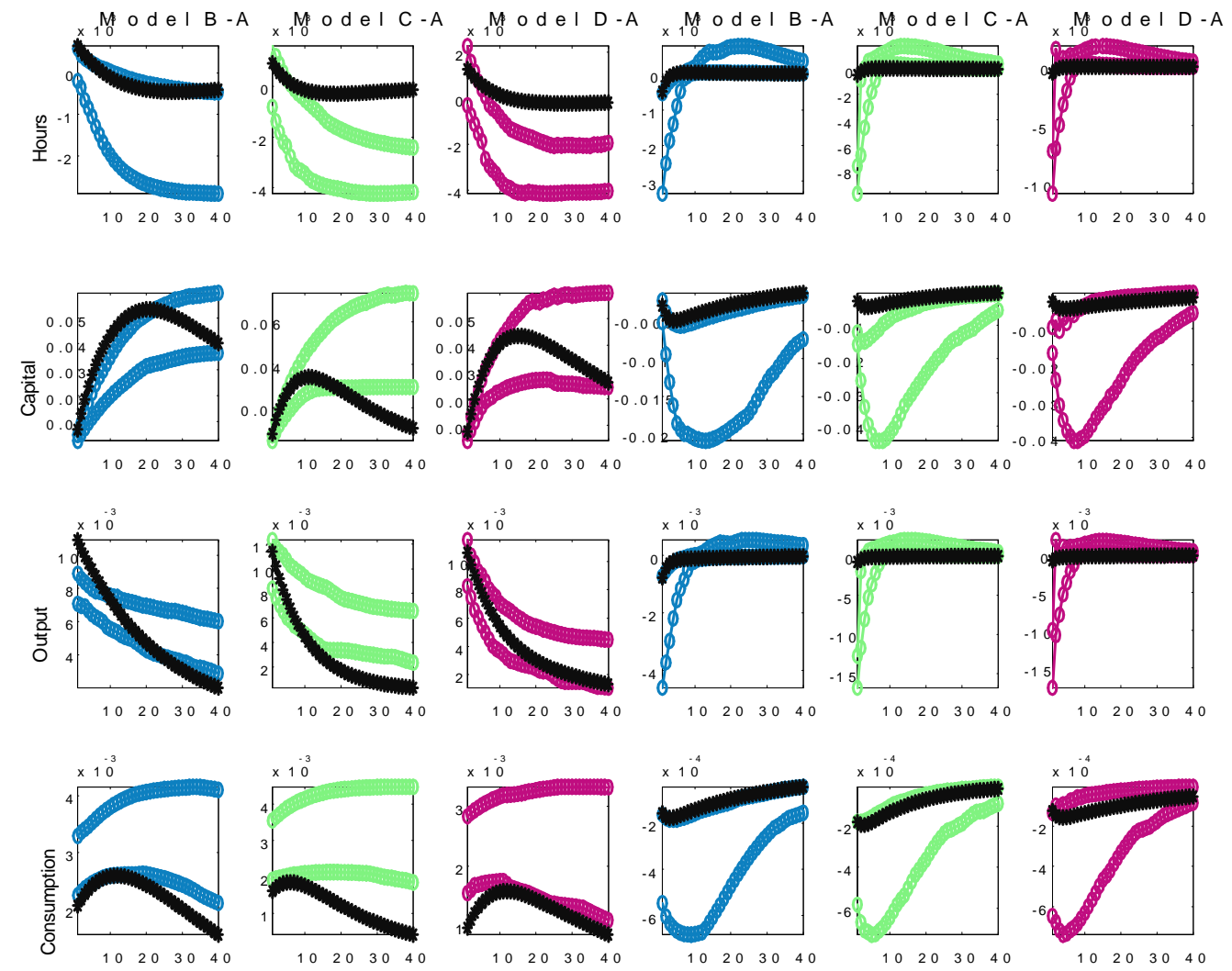

O-A84-A 16 *-B 50

$-\mathrm{A} 84-\mathrm{O}-\mathrm{A} 16-\mathrm{C} 5 \mathrm{P}$

$-{ }_{A} 84-0-A 16 * D 50$

Figure A3: Impulse responses, DGP2 (parameter variations explain 20 percent of output variance) 
small

\begin{tabular}{|l|c|c|c|c|}
\hline Variable & Technology & Government & Technology & \multicolumn{2}{c|}{ Government } \\
\hline & \multicolumn{2}{|c|}{ DGP: Model B } & \multicolumn{2}{|c|}{ Estimated: Time invariant } \\
\hline $\mathrm{Y}$ & 81.300 & 0.100 & 0.998 & 0.006 \\
$\mathrm{C}$ & 55.300 & 0.100 & 0.998 & 0.002 \\
$\mathrm{~N}$ & 15.600 & 0.400 & 0.978 & 0.025 \\
$\mathrm{~K}$ & 40.600 & 0.100 & 0.994 & 0.008 \\
\hline & \multicolumn{2}{|c|}{ DGP: Model C } & \multicolumn{2}{|c|}{ Estimated: Time invariant } \\
\hline $\mathrm{Y}$ & 81.900 & 0.100 & 0.927 & 0.082 \\
$\mathrm{C}$ & 26.500 & 0.100 & 0.999 & 0.001 \\
$\mathrm{~N}$ & 5.400 & 0.400 & 0.966 & 0.039 \\
$\mathrm{~K}$ & 37.400 & 0.100 & 0.974 & 0.030 \\
\hline & $\mathrm{DGP}:$ Model D & Estimated: Time invariant \\
\hline $\mathrm{Y}$ & 82.200 & 0.100 & 0.936 & 0.072 \\
$\mathrm{C}$ & 32.800 & 0.100 & 0.996 & 0.008 \\
$\mathrm{~N}$ & 10.200 & 0.500 & 0.928 & 0.079 \\
$\mathrm{~K}$ & 60.000 & 0.400 & 0.979 & 0.028 \\
\hline
\end{tabular}

Table A2: Variance decomposition, DGP2 (parameter variations explain 20 percent of output variance). 


\section{Appendix B : The RBC model with variable capital utilization of section 3}

The representative household maximizes the following stream of future utility

$$
\begin{aligned}
& \max E_{0} \sum_{j=0}^{\infty} \beta^{t} \frac{c_{t}^{1-\eta}}{1-\eta}-A \frac{n_{t}^{1+\gamma}}{1+\gamma} \\
c_{t}+i_{t}= & w_{t} n_{t}+r_{t}^{k} k_{t}^{s}-a\left(u_{t}\right) k_{t}^{s}-T_{t} \\
i_{t}= & k_{t}-(1-\delta) k_{t-1} \\
k_{t}^{s}= & u_{t} k_{t-1}
\end{aligned}
$$

where $c_{t}$ is consumption, $i_{t}$ investment, $k_{t}$ the stock of capital, and $n_{t}$ is hours worked. Household chooses the utilization rate of capital, $u_{t}$, and the amount of effective capital that she can rent to the firm, i.e. $k_{t}^{s}$. Household receives earnings from supplying labor and capital services to the firm, i.e. $w_{t}$ and $r_{t}^{k}$ respectively, subject to a cost of changing capital utilization, $a\left(u_{t}\right) k_{t}^{s}$. Finally, $T_{t}$ are lump sum taxes levied by the government. The production function is

$$
y_{t}=z_{t}\left(k_{t}^{s}\right)^{\alpha} n_{t}^{1-\alpha}
$$

A fraction of output is consumed by the government and financed with lump-sum taxes. The government budget constrain is always balanced, i.e.

$$
g_{t} y_{t}=T_{t}
$$

The optimality conditions of the planner problem are

$$
\begin{aligned}
\left(1-g_{t}\right) y_{t} & =c_{t}+k_{t}-\left(1-\delta-a\left(u_{t}\right) u_{t}\right) k_{t-1} \\
A n_{t}^{\gamma} c_{t}^{\eta} & =(1-\alpha)\left(1-g_{t}\right) y_{t} / n_{t} \\
1 & =\beta E_{t}\left(c_{t} / c_{t+1}\right)^{\eta}\left(1-\delta-a\left(u_{t+1}\right) u_{t+1}+\alpha\left(1-g_{t+1}\right) y_{t+1} / k_{t}\right) \\
\alpha\left(1-g_{t}\right) y_{t} / k_{t-1} & =u_{t}\left(a^{\prime}\left(u_{t}\right) u_{t}+a\left(u_{t}\right)\right) \\
y_{t} & =z_{t}\left(u_{t} k_{t-1}\right)^{\alpha} n_{t}^{1-\alpha} \\
a\left(u_{t}\right) & =\frac{1 / \beta+\delta-1}{\phi}\left(e^{\phi\left(u_{t}-1\right)}-1\right)
\end{aligned}
$$

The functional form for the adjustment cost of the capital utilization is in the last equation and it satisfies $a(1)=0, a^{\prime}(1)=1 / \beta+\delta-1, a^{\prime \prime}(1)=\phi(1 / \beta+\delta-1)$. If assume that, in the steady state $u=1$, the steady states for $\left(\frac{k}{y}, \frac{c}{y}, \frac{n}{y}, n\right)$ are the same as in the RBC model without variable capital utilization. 


\section{Appendix C : The equations of Gertler and Karadi model}

$$
\begin{aligned}
& \exp \left(\varrho_{t}\right)=\left(\exp \left(C_{t}\right)-h \exp \left(C_{t-1}\right)\right)^{-\sigma}-\beta h\left(\exp \left(C_{t+1}\right)-h \exp \left(C_{t}\right)\right)^{-\sigma} \\
& 1=\beta \exp \left(R_{t}\right) \exp \left(\Lambda_{t+1}\right) \\
& \exp \left(\Lambda_{t}\right)=\frac{\exp \left(\varrho_{t}\right)}{\exp \left(\varrho_{t-1}\right)} \\
& \chi * \exp \left(L_{t}\right)^{\varphi}=\exp \left(\varrho_{t}\right) \exp \left(P_{m, t}\right)(1-\alpha) \frac{\exp \left(Y_{t}\right)}{\exp \left(L_{t}\right)} \\
& \exp \left(\nu_{t}\right)=(1-\theta) \beta \exp \left(\Lambda_{t+1}\right)\left(\exp \left(R_{k, t+1}\right)-\exp \left(R_{t}\right)\right)+\beta \exp \left(\Lambda_{t+1}\right) \theta \exp \left(x_{t+1}\right) \exp \left(\nu_{t}(56)\right) \\
& \exp \left(\eta_{t}\right)=(1-\theta)+\beta \exp \left(\Lambda_{t+1}\right) \theta \exp \left(z_{t+1}\right) \exp \left(\eta_{t+1}\right) \\
& \exp \left(\phi_{t}\right)=\frac{1}{\left(1-\psi_{t}\right)} \frac{\exp \left(\eta_{t}\right)}{\lambda-\exp \left(\nu_{t}\right)} \\
& \exp \left(z_{t}\right)=\left(\exp \left(R_{k, t}\right)-\exp \left(R_{t-1}\right)\right)\left(1-\psi_{t-1}\right) \exp \left(\phi_{t-1}\right)+\exp \left(R_{t-1}\right) \\
& \exp \left(x_{t}\right)=\frac{\exp \left(\phi_{t}\right)\left(1-\psi_{t}\right)}{\left(\exp \left(\phi_{t-1}\right)\left(1-\psi_{t-1}\right)\right)} \exp \left(z_{t}\right) \\
& \exp \left(K_{t}\right)=\frac{\exp \left(\phi_{t}\right) \exp \left(N_{t}\right)}{\exp \left(Q_{t}\right)} \\
& \exp \left(N_{t}\right)=\exp \left(N e_{t}\right)+\exp \left(N n_{t}\right) \\
& \exp \left(N e_{t}\right)=\theta \exp \left(z_{t}\right) \exp \left(N_{t-1}\right) \exp \left(-e_{N e, t}\right)+\zeta_{t} \\
& \exp \left(N n_{t}\right)=\omega\left(1-\psi_{t-1}\right) \exp \left(Q_{t}\right) \exp \left(\xi_{t}\right) \exp \left(K_{t-1}\right) \\
& \exp \left(R_{k, t}\right)=\left(\exp \left(P_{m, t}\right) \alpha \frac{\exp \left(Y_{m, t}\right)}{\exp \left(K_{t-1}\right)}+\exp \left(\xi_{t}\right) *\left(\exp \left(Q_{t}\right)-\frac{\exp (\delta))}{\exp \left(Q_{t-1}\right)}\right.\right. \\
& \exp \left(Y_{m, t}\right)=\exp \left(a_{t}\right) *\left(\exp \left(\xi_{t}\right) * \exp \left(U_{t}\right) * \exp \left(K_{t-1}\right)\right)^{\alpha} * \exp \left(L_{t}\right)^{1-\alpha} \\
& \exp \left(Q_{t}\right)=1+0.5 \eta_{i}\left(\frac{\left(\operatorname{In}_{t}+I^{s}\right)}{\left(\operatorname{In}_{t-1}+I^{s}\right.}-1\right)^{2}+\eta_{i}\left(\frac{\left(\operatorname{In}_{t}+I^{s}\right)}{\left(\operatorname{In}_{t-1}+I^{s}\right.}-1\right) \frac{\left(\operatorname{In}_{t}+I^{s}\right)}{\left(\operatorname{In}_{t-1}+I^{s} s\right)} \\
& -\beta \exp \left(\Lambda_{t+1}\right) \eta_{i}\left(\frac{\left(\operatorname{In}_{t+1}+I^{s}\right)}{\left(\operatorname{In}_{t}+I^{s}\right.}-1\right)\left(\frac{\left(\operatorname{In}_{t+1}+I^{s}\right)}{\left(\operatorname{In}_{t}+I^{s}\right)}\right)^{2} \\
& \exp (\delta)=\delta_{c}+b /(1+\zeta) * \exp \left(U_{t}\right)^{1+\zeta} \\
& \alpha \frac{\exp \left(Y_{m}\right)}{\exp \left(U_{t}\right)}=\frac{b \exp \left(U_{t}\right)^{\zeta} \exp \left(\xi_{t}\right) * \exp \left(K_{t-1}\right)}{\exp \left(P_{m, t}\right)} \\
& I n_{t}=\exp \left(I_{t}\right)-\exp (\delta) * \exp \left(\xi_{t}\right) * \exp \left(K_{t-1}\right) \\
& \exp \left(K_{t}\right)=\exp \left(\xi_{t}\right) * \exp \left(K_{t-1}\right)+I n_{t} \\
& \exp \left(G_{t}\right)=G^{s} * \exp \left(g_{t}\right) \\
& \exp \left(Y_{t}\right)=\exp \left(C_{t}\right)+\exp \left(G_{t}\right)+\exp \left(I_{t}\right)+0.5 \eta_{i}\left(\frac{\left(I n_{t}+I^{s}\right)}{\left(I n_{t-1}+I^{s}\right.}-1\right)^{2}\left(I n_{t}+I^{s}\right)+\tau \psi \exp \left(\left(\boldsymbol{K}_{\boldsymbol{\theta}}\right)\right. \\
& \exp \left(Y_{m, t}\right)=\exp \left(Y_{t}\right) * \exp \left(D_{t}\right)
\end{aligned}
$$




$$
\begin{aligned}
\exp \left(D_{t}\right) & =\gamma * \exp \left(D_{t-1}\right) * \exp \left(i n f l_{t-1}\right)^{-\gamma_{P} * \epsilon} \exp \left(i n f l_{t}\right)^{\epsilon} \\
& +(1-\gamma)\left(\left(1-\gamma \exp \left(i n f l_{t-1}\right)^{\gamma_{P}(1-\gamma)} \exp \left(i n f l_{t}\right)^{\gamma-1}\right) /(1-\gamma)\right)^{-\epsilon /(1-\gamma)} \\
\exp \left(X_{t}\right) & =1 / \exp \left(P_{m, t}\right) \\
\exp \left(F_{t}\right) & =\exp \left(Y_{t}\right) * \exp \left(P_{m, t}\right)+\beta \gamma \exp \left(\Lambda_{t+1}\right) \exp \left(i n f l_{t+1}\right)^{\epsilon}\left(\exp \left(i n f l_{t}\right)\right)^{-\epsilon \gamma_{P}} \exp (F(t 77) \\
\exp \left(Z_{t}\right) & =\exp \left(Y_{t}\right)+\beta \gamma \exp \left(\Lambda_{t+1}\right) \exp \left(i n f l_{t+1}\right)^{\epsilon-1} \exp \left(i n f l_{t}\right)^{\gamma_{P} *(1-\epsilon)} \exp \left(Z_{t+1}\right) \\
\exp \left(i n f l_{t}^{*}\right) & =\frac{\epsilon}{\epsilon-1} \frac{\exp \left(F_{t}\right)}{\exp \left(Z_{t}\right)} \exp \left(i n f l_{t}\right) \\
\left(\exp \left(i n f l_{t}\right)\right)^{1-\epsilon} & =\gamma \exp \left(i n f l_{t-1}\right)^{\gamma_{P}(1-\epsilon)}+(1-\gamma)\left(\exp \left(i n f l_{t}^{*}\right)\right)^{1-\epsilon} \\
\exp \left(i_{t}\right) & =\exp \left(R_{t}\right) * \exp \left(i n f l_{t+1}\right) \\
\exp \left(i_{t}\right) & =\exp \left(i_{t-1}\right)^{\rho_{i}}\left(\beta^{-1} \exp \left(i n f l_{t}\right)^{\kappa_{\pi}} *\left(\exp \left(X_{t}\right) /(\epsilon /(\epsilon-1))\right)^{\kappa_{y}}\right)^{1-\rho_{i}} \exp \left(e_{i, t}\right) \\
\psi_{t} & =\kappa *\left(R_{k, t+1}-R_{t}-R_{k}^{s}+R^{s}\right)+e_{\psi, t} \\
a_{t} & =\rho_{a} * a_{t-1}-\sigma_{a} * e_{a, t} \\
\xi_{t} & =\rho_{\xi} * \xi_{t-1}-\sigma_{\xi} * e_{\xi, t} \\
g_{t} & =\rho_{g} * g_{t-1}-e_{g, t} \\
e_{\psi, t} & =\rho_{\psi} * e_{\psi, t-1}+e_{\psi, t} \\
\zeta_{t} & =\rho_{\zeta} * \zeta_{t-1}+e_{\zeta, t}
\end{aligned}
$$

Note: $\zeta_{t}=0, \forall t$ in the basic estimated model. It appears only in the augmented model of table 7 . 
Centre for Applied Macro - and Petroleum economics (CAMP)

will bring together economists working on applied macroeconomic issues, with special emphasis on petroleum economics.

BI Norwegian Business School

Centre for Applied Macro - Petroleum economics (CAMP)

N-0442 Oslo

http://www.bi.no/camp 